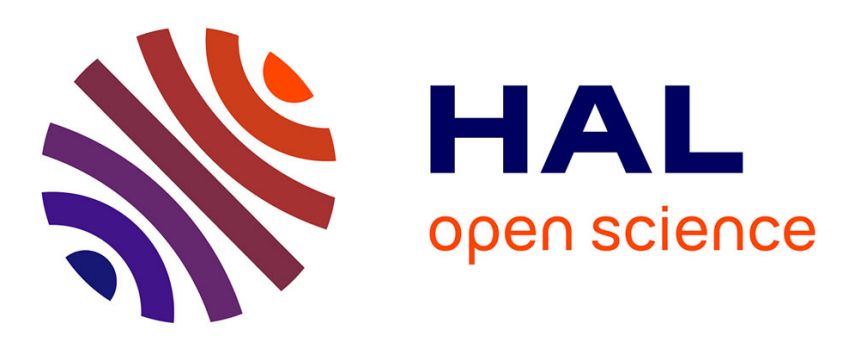

\title{
Nonlinear vibratory energy exchanges between a two degrees-of-freedom pendulum and a nonlinear absorber
} Gabriel Hurel, Alireza Ture Savadkoohi, Claude-Henri Lamarque

\section{To cite this version:}

Gabriel Hurel, Alireza Ture Savadkoohi, Claude-Henri Lamarque. Nonlinear vibratory energy exchanges between a two degrees-of-freedom pendulum and a nonlinear absorber. Journal of Engineering Mechanics - ASCE, 2019, 145 (8), pp.04019058. 10.1061/(ASCE)EM.1943-7889.0001620 . hal-01993589

\section{HAL Id: hal-01993589 \\ https://hal.science/hal-01993589}

Submitted on 5 Jun 2019

HAL is a multi-disciplinary open access archive for the deposit and dissemination of scientific research documents, whether they are published or not. The documents may come from teaching and research institutions in France or abroad, or from public or private research centers.
L'archive ouverte pluridisciplinaire HAL, est destinée au dépôt et à la diffusion de documents scientifiques de niveau recherche, publiés ou non, émanant des établissements d'enseignement et de recherche français ou étrangers, des laboratoires publics ou privés. 


\title{
Nonlinear vibratory energy exchanges between a two degrees-of-freedom pendulum and a nonlinear absorber.
}

\author{
G. Hurel, A. Ture Savadkoohi, C.-H. Lamarque \\ Univ. Lyon, ENTPE, LTDS UMR 5513, rue Maurice Audin, 69518 Vaulx-en-Velin \\ Email: gabriel.hurel@entpe.fr
}

Accepted on December 5, 2018

\begin{abstract}
The multi-time scale responses of a two degrees of freedom pendulum coupled with a nonlinear absorber is studied. The absorber is positioned in an arbitrary direction with respect to those of the pendulum oscillations. Phase-dependent slow invariant manifold of the system and its stable zones are traced at fast time scale while studying system responses at slow time scale around the slow invariant manifold leads to detection of equilibrium and singular points. Moreover, the amplitude-frequency curves of the system are detected showing the possibility of existence of isolated branches, which could correspond to high energy levels for pendulum oscillations. All analytic developments are confronted with numerical results collected from direct numerical integration of system equations. Depending on characteristics of external excitations, the system can face periodic or modulated regimes.
\end{abstract}

Keywords pendulum, damper, nonlinear dynamics, nonlinear energy sink, multiscale method, passive control

\section{Introduction}

Design of structural systems against vibrations induced by internal and external excitations such as inter-particle actions, nature, aerodynamic forces, parametric excitations, etc, seeks for robust and reliable controller devices. The control process of structures covers large variety of systems ranging from active to passive devices [1]. The most widely used control devices are passive systems which do not need any external sources for their activations [2]. Meanwhile, the passive control systems are categorized into linear and nonlinear ones: Frahm device [3] or tuned mass dampers (TMD) [4] are linear passive systems. Here, the term "linear" stands for the linear restoring forcing function of the coupled oscillator, i.e. the controller, which reads as $F(\alpha)=K_{l} \alpha$. The variable $\alpha$ is the generalized displacement while $K_{l}$ is the relevant stiffness of the system which is responsible for its linear behaviors. If such systems are tuned well versus the linear frequency of main structures, they are able to control vibratory energy of main structures. Apart from being heavy, the main drawback of such systems is that they are not efficient for a large frequency width while they modify the frequency of the system.

Roberson [5] presented a system which its restoring forcing function reads as $F(\alpha)=K_{l} \alpha+$ $K_{n l} \alpha^{3}$ where $K_{n l}$ stands for the rigidity of the system which imposes cubic nonlinearity to its restoring forcing function. They showed that via exploiting the nonlinear capacities of the coupled controller system, the suppression band of vibratory energy would increase and the controller would be operational for wide frequency ranges. Since then, many nonlinear 


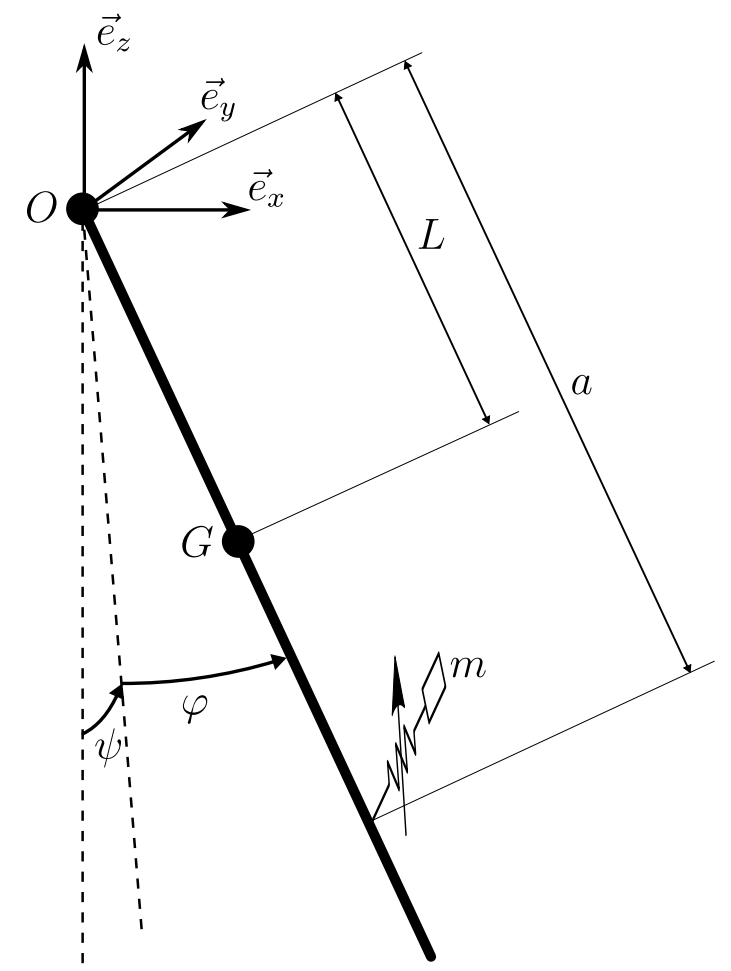

Figure 1: The pendulum with the center of mass $G$ rotates in longitudinal (with the angle $\varphi$ ) and transversal (with the angle $\psi$ ) directions. A NES with the mass $m$ is coupled to the system at the distance $a$ from the base of the pendulum $O$.

passive systems have been developed. One of them, which is of the interest of the current paper, is named as nonlinear energy sink (NES) $[6,7]$ which contains pure nonlinear restoring forcing function (with no linear part) $[8,9]$. The control process by the NES systems is based on the nonlinear interactions between main oscillators and the NES followed by bifurcation(s) leading to periodic or strongly modulated regimes (SMR) [10]. The aim of the current paper is to control oscillations of a two degrees of freedom (dof) pendulum.

The control process of a single dof pendulum, based on small rotations assumptions by a TMD is carried out by Matsuhisa et al. [11, 12]. The passive control of a two-dof system, which can be represented by the small oscillations of a two-dof pendulum, by a NES coupled to the second dof is studied by Starosvetsky and Gendelman [13] and Pham et al. [14].

The current manuscript aims at studying vibratory energy exchanges between a two-dof pendulum which is coupled to a NES. The goal is to analyse the possibility of the passive control of the pendulum oscillations via a NES positioned at a given direction. Organization of the paper is as it follows: First of all, governing system equations considering a semi-rigid constraint for the pendulum at one direction is provided. The complexified version of system variables are treated by a multiple scale technique to detect its slow invariant manifold, equilibrium and singular points. The paper is followed by some numerical examples and discussions on the system with coupled NES with cubic and non-smooth nonlinearities. Finally the paper is concluded. 


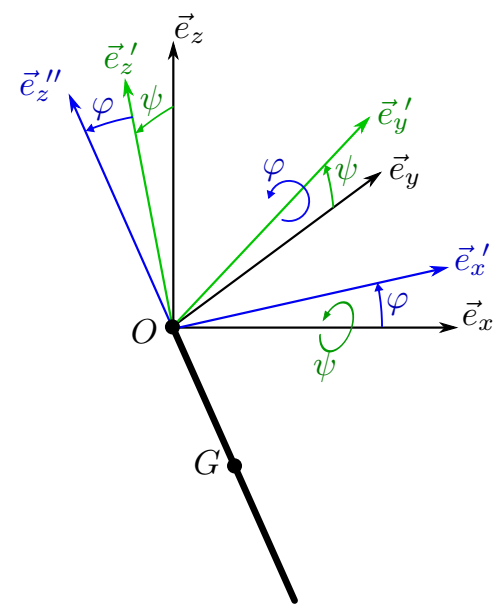

Figure 2: Rotational transformations of reference frames.

\section{Studied system}

\subsection{The main system}

A pendulum shown in Fig. 1, maintained at point $O$ can oscillate in $\vec{e}_{x}$ and $\vec{e}_{y}$ directions of the main reference frame $\left(\vec{e}_{x}, \vec{e}_{y}, \vec{e}_{z}\right)$. As seen on Fig. 2, the main reference frame is transformed by the rotation of an angle denoted as $\psi$ around the $\vec{e}_{x}$ axis to a secondary reference frame $\left(\vec{e}_{x}, \vec{e}_{y}^{\prime}, \vec{e}_{z}^{\prime}\right)$. A second rotation of an angle denoted as $\varphi$ around the $\vec{e}_{y}^{\prime}$ axis transforms to the secondary reference frame in the local reference frame of the pendulum $\left(\vec{e}_{x}^{\prime}, \vec{e}_{y}^{\prime}, \vec{e}_{z}^{\prime \prime}\right)$.

The pendulum has a mass $M$, and its inertia tensor is noted $\mathbf{I}$ in its local reference frame $\left(\vec{e}_{x}^{\prime}, \vec{e}_{y}^{\prime}, \vec{e}_{z}^{\prime \prime}\right)$. The pendulum has two planes of symmetry so that the products of inertia $I_{x y}, I_{x z}$ and $I_{y z}$ are zero. We suppose that the rotational stiffness $K_{y}$ exists only around the $\vec{e}_{x}$ axis which creates a semi-rigid joint around $\vec{e}_{x}$. Its center of mass $G$ is located on the axis $\vec{e}_{z}^{\prime \prime}$ at a distance $L$ from the point $O$ as seen on Fig. 1 .

The coordinates of the center of mass $G$ in the main reference frame read:

$$
\left\{\begin{array}{l}
x_{G}=L \sin (\varphi) \\
y_{G}=L \sin (\psi) \cos (\varphi) \\
z_{G}=-L \cos (\varphi) \cos (\psi)
\end{array}\right.
$$

The pendulum is excited by a sinusoidal generalized force of amplitude $f_{0}$ and frequency $\Omega$. This force is applied around the axis $\vec{e}_{f}$ in the plane $\left(\vec{e}_{x}, \vec{e}_{y}\right)$ forming a angle $\beta$ with the axis $\vec{e}_{y}$ as shown on Fig. 3b.

\subsection{Coupled NES}

To meet the industrial constraints, only a single NES is attached to the system. It is coupled to the main system at a distance $a$ from the point $O$ on the line $O G$ on the orthogonal direction to $O G$ (see Fig. 1). The restoring forcing function of the NES is characterized by a function $S(u)$, where $u$ stands for the relative displacement of the mass $m$.

The direction of the NES with the mass $m$ is determined by a constant angle $\alpha \in\left[0, \frac{\pi}{2}\right]$ with the axis $\vec{e}_{x}^{\prime}$ as shown in Fig. 3b. In the local reference frame, the coordinates of the mass $m$ of the NES read:

$$
\left\{\begin{array}{l}
x_{m}^{\prime}=u \cos (\alpha) \\
y_{m}^{\prime}=u \sin (\alpha) \\
z_{m}^{\prime \prime}=-a
\end{array}\right.
$$




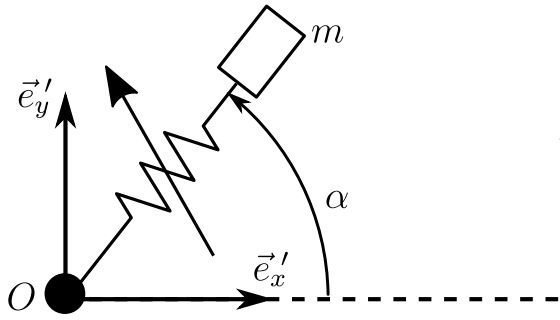

(a) Angle $\alpha$

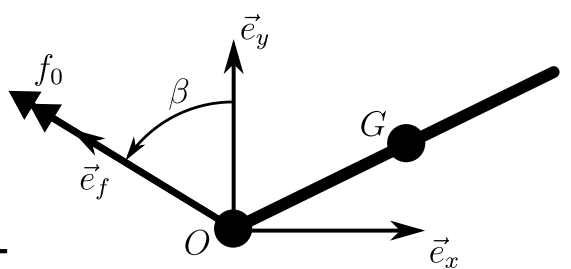

(b) Angle $\beta$

Figure 3: The orientations of the NES and applied generalized external excitation: (a) The angle $\alpha$ representing the location of the NES. (b) The pendulum is under generalized external excitation around axis $\vec{e}_{f}$ with the amplitude $f_{0} . \beta$ represents the angle between $\vec{e}_{f}$ and $\vec{e}_{y}$.

In the main reference frame, these coordinates become:

$$
\left\{\begin{array}{l}
x_{m}=a \sin (\varphi)+u \cos (\varphi) \cos (\alpha) \\
y_{m}=a \sin (\psi) \cos (\varphi)+u(\cos (\psi) \sin (\alpha)-\sin (\varphi) \sin (\psi) \cos (\alpha)) \\
z_{m}=-a \cos (\varphi) \cos (\psi)+u[\sin (\varphi) \cos (\psi) \cos (\alpha)+\sin (\psi) \sin (\alpha)]
\end{array}\right.
$$

\subsection{Governing system equations}

The kinetic $(\mathcal{K})$ and potential $(\mathcal{U})$ energies of the mechanical system read:

$$
\left\{\begin{array}{l}
\mathcal{K}=\frac{1}{2} M\left(\dot{x}_{G}^{2}+\dot{y}_{G}^{2}+\dot{z}_{G}^{2}\right)+\frac{1}{2} I_{y y} \dot{\varphi}^{2}+\frac{1}{2} I_{x x} \dot{\psi}^{2}+\frac{1}{2} m\left(\dot{x}_{m}^{2}+\dot{y}_{m}^{2}+\dot{z}_{m}^{2}\right) \\
\mathcal{U}=g\left(M z_{G}+m z_{m}\right)+\frac{1}{2} K_{y} \psi^{2}+\mathcal{S}(u)
\end{array}\right.
$$

where $\nabla \mathcal{S}(u)=S(u)$. The damping and external forces of the system read:

$$
\begin{aligned}
& F_{\varphi}=-C_{x} \dot{\varphi}+\cos (\beta) f_{0} \sin (\Omega t) \\
& F_{\psi}=-C_{y} \dot{\psi}+\sin (\beta) f_{0} \sin (\Omega t) \\
& F_{u}=-C_{u} \dot{u}
\end{aligned}
$$

By applying the Lagrange equations, the system equations of the system read:

$$
\begin{aligned}
& {\left[L^{2}+j_{x}+\varepsilon\left(a^{2}+u^{2} \cos ^{2}(\alpha)\right)\right] \ddot{\varphi}+\varepsilon\left[\sin (\varphi) a u+\cos (\alpha) \cos (\varphi) u^{2}\right] \sin (\alpha) \ddot{\psi}} \\
& \quad+a \varepsilon \cos (\alpha) \ddot{u}+\varepsilon c_{x} \dot{\varphi}+\left[\sin (\varphi) L^{2} \cos (\varphi)+\varepsilon \sin (\varphi) a^{2} \cos (\varphi)-\varepsilon a u \cos (\alpha)\right. \\
& \left.\quad+2 \varepsilon a \cos (\alpha) \cos ^{2}(\varphi) u-\varepsilon \sin (\varphi) u^{2} \cos ^{2}(\alpha) \cos (\varphi)\right] \dot{\psi}^{2} \\
& \quad+\varepsilon[2 a \sin (\alpha) \sin (\varphi)+2 \sin (\alpha) \cos (\alpha) \cos (\varphi) u] \dot{\psi} \dot{u}+2 \varepsilon \cos ^{2}(\alpha) u \dot{\varphi} \dot{u} \\
& \quad+g[L \sin (\varphi) \cos (\psi)+\varepsilon(a \sin (\varphi) \cos (\psi)+\cos (\alpha) \cos (\varphi) \cos (\psi) u)] \\
& \quad-\varepsilon f_{0} \cos (\beta) \sin (\Omega t)=0
\end{aligned}
$$




$$
\begin{aligned}
& {\left[j_{y}+L^{2} \cos ^{2}(\varphi)+\varepsilon\left(u^{2}-u^{2} \cos ^{2}(\alpha) \cos ^{2}(\varphi)-2 a u \cos (\alpha) \cos (\varphi) \sin (\varphi)\right.\right.} \\
& \left.\left.\quad+a^{2} \cos ^{2}(\varphi)\right)\right] \ddot{\psi}+\left[\varepsilon u^{2} \cos (\alpha) \cos (\varphi) \sin (\alpha)+a \varepsilon u \sin (\alpha) \sin (\varphi)\right] \ddot{\varphi} \\
& \quad+a \varepsilon \cos (\varphi) \sin (\alpha) \ddot{u}+c_{y} \dot{\psi} \varepsilon+\left[a \varepsilon u \cos (\varphi) \sin (\alpha)-\varepsilon u^{2} \cos (\alpha) \sin (\alpha) \sin (\varphi)\right] \dot{\varphi}^{2} \\
& \quad+\left[-2 L^{2} \cos (\varphi) \sin (\varphi)-2 \varepsilon\left(a^{2} \cos (\varphi) \sin (\varphi)+a u \cos (\alpha)\right.\right. \\
& \left.\left.\quad+u^{2} \cos ^{2}(\alpha) \cos (\varphi) \sin (\varphi)-2 a u \cos (\alpha) \cos ^{2}(\varphi)\right)\right] \dot{\varphi} \dot{\psi}+2 \varepsilon u \cos (\alpha) \cos (\varphi) \sin (\alpha) \dot{\varphi} \dot{u} \\
& \quad+2 \varepsilon\left[u-a \cos (\alpha) \cos (\varphi) \sin (\varphi)-u \cos ^{2}(\alpha) \cos ^{2}(\varphi)\right] \dot{\psi} \dot{u} \\
& \quad+g[L \cos (\varphi) \sin (\psi)+\varepsilon(a \cos (\varphi) \sin (\psi) \\
& \quad+u \cos (\psi) \sin (\alpha)-u \cos (\alpha) \sin (\varphi) \sin (\psi))]+k_{y} \psi-\varepsilon f_{0} \sin \beta \sin (\Omega t)=0 \\
& \varepsilon[a \cos (\alpha) \ddot{\varphi}+a \cos (\varphi) \sin (\alpha) \ddot{\psi}+\ddot{u}+\lambda \dot{u}+(a \cos (\alpha) \cos (\varphi) \sin (\varphi) \\
& \left.\quad-u+u \cos ^{2}(\alpha) \cos ^{2}(\varphi)\right) \dot{\psi}^{2}-2(a \sin (\alpha) \sin (\varphi)+u \cos (\alpha) \cos (\varphi) \sin (\alpha)) \dot{\varphi} \dot{\psi} \\
& \left.\quad-\dot{\varphi}^{2} u \cos ^{2}(\alpha)+g(\sin (\alpha) \sin (\psi)+\cos (\alpha) \cos (\psi) \sin (\varphi))\right]+s(u)=0
\end{aligned}
$$

where $\varepsilon=\frac{m}{M}, j_{x}=\frac{I_{y y}}{M}, j_{y}=\frac{I_{x x}}{M}, c_{x}=\frac{C_{x}}{m}, c_{y}=\frac{C_{y}}{m}, \lambda=\frac{C_{u}}{m}, k_{y}=\frac{K_{y}}{M}$ and $s(u)=$ $\frac{S(u)}{M}$. It should be underlined that the parameter $\varepsilon$ is very small, i.e. $0<\varepsilon \ll 1$. For every simulations and examples, the default values of parameters are: $j_{x}=j_{y}=10 \mathrm{~m}^{2}$, $k_{y}=0.1 \mathrm{~s}^{-2}, g=9.81 \mathrm{~m} \mathrm{~s}^{-2}, L=a=1 \mathrm{~m}, \lambda=0.1 \mathrm{~s}^{-1}, c_{x}=c_{y}=50 \mathrm{~s}^{-1}$ and $\alpha=\frac{\pi}{6}$.

\section{Analytical study}

For the analytical study we assume that $\varphi, \psi$ and $u$ are small. These variables are rescaled with the parameter $\varepsilon$ :

$$
\varphi=\varepsilon \tilde{\varphi}, \quad \psi=\varepsilon \tilde{\psi}, \quad u=\varepsilon \tilde{u}
$$

We consider also a small excitation amplitude via setting $f_{0}=\varepsilon \tilde{f}_{0}$.

In Eqs. 8, 9 and 10, the sine and cosine functions are expressed in the form of series as:

$$
\sin (\varepsilon \tilde{\varphi})=\sum_{k=0}^{\infty} \varepsilon^{2 k+1} \frac{\tilde{\varphi}^{2 k+1}(-1)^{k}}{(2 k+1) !}, \quad \cos (\varepsilon \tilde{\varphi})=\sum_{k=0}^{\infty} \varepsilon^{2 k} \frac{\tilde{\varphi}^{2 k}(-1)^{k}}{2 k !}
$$

The complex variables of Manevitch [15] are introduced to system variables as:

$$
\Phi e^{i \Omega t}=\dot{\tilde{\varphi}}+i \Omega \tilde{\varphi}, \quad \Psi e^{i \Omega t}=\dot{\tilde{\psi}}+i \Omega \tilde{\psi}, \quad U e^{i \Omega t}=\dot{\tilde{u}}+i \Omega \tilde{u}
$$

where $i^{2}=-1$.

In the following, we will use a multiple scale method [16] to treat system equations. We consider that time $t$ is embedded in several scales linked to each other with the parameter $\varepsilon$ as:

$$
\tau_{0}=t, \tau_{1}=\varepsilon^{1} t, \tau_{2}=\varepsilon^{2} t, \ldots
$$

Time $\tau_{0}$ corresponds to fast time whereas $\tau_{1}, \tau_{2}, \ldots$ are slow time scales. The derivation operator is redefined with the different time scales:

$$
\frac{\mathrm{d}}{\mathrm{d} t}=\frac{\partial}{\partial \tau_{0}}+\varepsilon \frac{\partial}{\partial \tau_{1}}+\varepsilon^{2} \frac{\partial}{\partial \tau_{2}}+\ldots
$$

Theoretically, the response of the system includes several harmonics. In order to consider only the first harmonics, a Galerkin method is used. This can be done for an arbitrary function $f\left(\tau_{0}, \tau_{1}, \tau_{2}, \ldots\right)$ of the system via:

$$
F=\frac{\Omega}{2 \pi} \int_{0}^{\frac{2 \pi}{\Omega}} f\left(\tau_{0}, \tau_{1}, \tau_{2}, \ldots\right) e^{-i \Omega \tau_{0}} \mathrm{~d} \tau_{0}
$$


In applying Eq. 16, we will suppose that $\Phi, \Psi$ and $U$ are independent of time $\tau_{0}$. This will be verified during the multiple scale method or we will search for an asymptotic behavior when $\tau_{0} \longrightarrow \infty$.

In the following sections we will consider system responses at different scales of time which is equivalent to consider system equations at different order of $\varepsilon$. Moreover, we assume both natural frequencies of the main system are very close to each other, i.e.:

$$
\omega_{1}=\omega_{2}+\eta \varepsilon
$$

where $\omega_{1}$ and $\omega_{2}$ are the natural frequencies of the main system around $\vec{e}_{y}$ and $\vec{e}_{x}$ axes:

$$
\omega_{1}^{2}=\frac{L g}{j_{x}+L^{2}} \quad \omega_{2}^{2}=\frac{L g+k_{y}}{j_{y}+L^{2}}
$$

Moreover, we consider a special case where the frequency of the excitation $\Omega$ is close to both natural frequencies of the main system:

$$
\Omega=\omega_{j}+\sigma_{j} \varepsilon \text { for } j=[1,2]
$$

In the remainder of the article, considering the Eq. 17, $j$ can be assigned to the value 1 or 2. For the sake of simplicity, the frequency $\omega_{j}$ is noted $\omega$.

\subsection{Fast time scale, i.e. $\varepsilon^{0}$ order of the system equations}

With the operations described previously, the equations of the system at $\tau_{0}$ time scale read:

$$
\left\{\begin{array}{l}
\frac{\partial \Phi}{\partial \tau_{0}}=0 \\
\frac{\partial \Psi}{\partial \tau_{0}}=0 \\
a \cos (\alpha) \frac{\partial \Phi}{\partial \tau_{0}}+a \sin (\alpha) \frac{\partial \Psi}{\partial \tau_{0}}+\frac{\partial U}{\partial \tau_{0}}+i \cos (\alpha) \frac{a \Omega^{2}-g}{2 \Omega} \Phi \\
\quad+i \sin (\alpha) \frac{a \Omega^{2}-g}{2 \Omega} \Psi+\frac{i \Omega+\lambda}{2} U+G(U)=0
\end{array}\right.
$$

The $G(U)$ function is obtained via application of the Eq. 16 on the nonlinear restoring forcing function of the system. If $S(u)=k_{c} u^{3}$ then:

$$
G(U)=-\frac{3 i \tilde{k}_{\mathrm{c}}}{8 \omega^{3}} U|U|^{2}
$$

where $\tilde{k}_{c}=k_{c} \varepsilon$. In the rest of the article and for numerical results, we take $\tilde{k}_{\mathrm{c}}=1 \mathrm{~m}^{-2} \mathrm{~s}^{-2}$.

\subsubsection{Slow invariant manifold of the system}

From the Eqs. 20 and 21 we conclude $\Phi$ and $\Psi$ are independent of fast time scale. In this case, the Eq. 22 becomes:

$$
\frac{\partial U}{\partial \tau_{0}}+i \cos (\alpha) \frac{a \omega^{2}-g}{2 \omega} \Phi+i \sin (\alpha) \frac{a \omega^{2}-g}{2 \omega} \Psi+\frac{i \omega+\lambda}{2} U+G(U)=0
$$

Let us seek for an asymptotic state when $\tau_{0} \longrightarrow \infty$. This means that we are interested to trace system behavior at its fixed points. Eq. 24 reads:

$$
i \cos (\alpha) \frac{a \omega^{2}-g}{2 \omega} \Phi+i \sin (\alpha) \frac{a \omega^{2}-g}{2 \omega} \Psi+\frac{i \omega+\lambda}{2} U+G(U)=0
$$


The complex variables can be rewritten in polar form as $\Phi=N_{\varphi} e^{i \delta_{\varphi}}, \Psi=N_{\psi} e^{i \delta_{\psi}}$ and $U=N_{u} e^{i \delta_{u}}$. With a cubic function of the NES, the Eq. 25 yield to:

$$
\frac{3 \tilde{k}_{\mathrm{c}}}{4 \omega^{2}} N_{u}^{3}-\omega^{2} N_{u}-i \omega \lambda N_{u}=\left(a \omega^{2}-g\right)\left(\cos (\alpha) N_{\varphi} e^{i\left(\delta_{\varphi}-\delta_{u}\right)}+\sin (\alpha) N_{\psi} e^{i\left(\delta_{\psi}-\delta_{u}\right)}\right)
$$

Via separating real and imaginary parts of the Eq. 26, we obtain:

$$
\left\{\begin{array}{r}
-\lambda \omega N_{u}=\left(a \omega^{2}-g\right)\left[\cos (\alpha) N_{\varphi} \sin \left(\delta_{\varphi}-\delta_{u}\right)+\sin (\alpha) N_{\psi} \sin \left(\delta_{\psi}-\delta_{u}\right)\right] \\
\frac{3 \tilde{k}_{\mathrm{c}}}{4 \omega^{2}} N_{u}^{3}-\omega^{2} N_{u}=\left(a \omega^{2}-g\right)\left[\cos (\alpha) N_{\varphi} \cos \left(\delta_{\varphi}-\delta_{u}\right)+\sin (\alpha) N_{\psi} \cos \left(\delta_{\psi}-\delta_{u}\right)\right]
\end{array}\right.
$$

SO

$$
\mathcal{H}=\cos ^{2}(\alpha) N_{\varphi}^{2}+\sin ^{2}(\alpha) N_{\psi}^{2}+2 N_{\varphi} N_{\psi} \sin (\alpha) \cos (\alpha) \cos (\delta)-P^{2}\left(N_{u}\right)=0
$$

where

$$
P^{2}\left(N_{u}\right)=\frac{\left(\frac{3 \tilde{k}_{\mathrm{c}}}{4 \omega^{2}} N_{u}^{3}-\omega^{2} N_{u}\right)^{2}+\lambda^{2} \omega^{2} N_{u}^{2}}{\left(a \omega^{2}-g\right)^{2}} \quad \text { and } \quad \delta=\delta_{\varphi}-\delta_{\psi}
$$

Eq. 28 corresponds to the slow invariant manifold (SIM) of the system that is a geometrical surface for all system behaviors, including periodic [17] and strongly modulated responses [18]. The SIM is plotted in Fig. 4 for several values of $\delta$.

A special case happens when $N_{\varphi}=0$ or $N_{\psi}=0$. Let us consider the case when $N_{\psi}=0$, the Eq. 28 becomes:

$$
N_{\varphi}=\frac{P\left(N_{u}\right)}{\cos (\alpha)}
$$

In this case, the SIM becomes independent of the phase $\delta$.

The phase $\delta$ is involved only when $N_{\varphi}$ and $N_{\psi}$ are nonzero, i.e. when the pendulum is activated in both directions. For a given value of $P\left(N_{u}\right)$, the amplitudes $N_{\varphi}$ and $N_{\psi}$ describe a parabola if:

$$
\cos ^{2}(\delta)<1
$$

This case is illustrated in Figs. 4b and $4 \mathrm{c}$ for two values of phase $\delta$. The Eq. 31 is false when $\delta=k \pi, k \in \mathbb{Z}$. Two cases are possible:

- If $k$ is an even number, the Eq. 28 becomes:

$$
\cos (\alpha) N_{\varphi}+\sin (\alpha) N_{\psi}=P\left(N_{u}\right)
$$

For a given value of $N_{u}$, the variables $N_{\varphi}$ and $N_{\psi}$ describe a line as shown on Fig. 4a.

- If $k$ is an odd number, the Eq. 28 becomes:

$$
\cos (\alpha) N_{\varphi}-\sin (\alpha) N_{\psi}= \pm P\left(N_{u}\right)
$$

which indicates that for a given value of $N_{u}$, the variables $N_{\varphi}$ and $N_{\psi}$ describe two lines.

In this case, as shown on Fig. $4 \mathrm{~d}$, the SIM is degenerate and if $N_{\varphi}=\tan (\alpha) N_{\psi}$, then $N_{u}=0$. Thus, the NES is decoupled from the main system.

The phase $\delta=k \pi$ where $k$ is an odd number means the angles $\varphi$ and $\psi$ of the pendulum are in phase opposition (see Eq. 29). Thus, the path of the point $G$ of the pendulum projected on the plane $\left(\vec{e}_{x}, \vec{e}_{y}\right)$ is a straight line. The decoupling of the NES happens when the trajectory of the point $G$ is orthogonal to the positioning direction of the NES i.e. $N_{\varphi}=\tan (\alpha) N_{\psi}$.

This issue will be treated in the numerical study. 


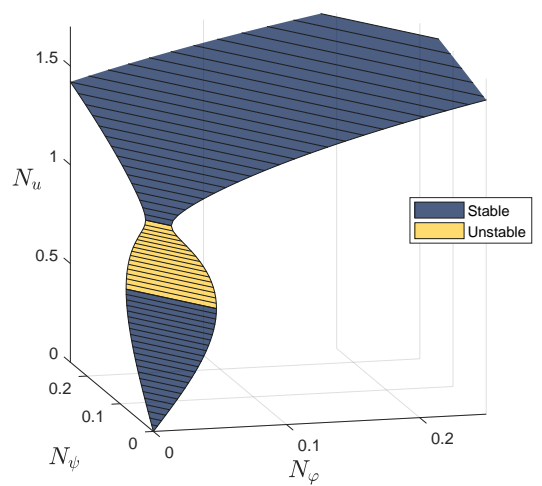

(a) $\delta=0$

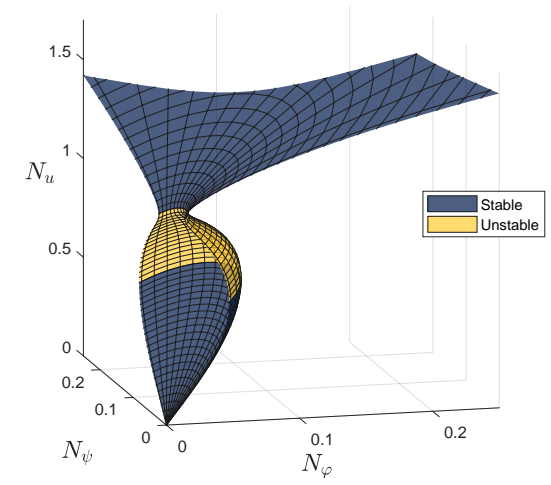

(c) $\delta=0.8 \pi$

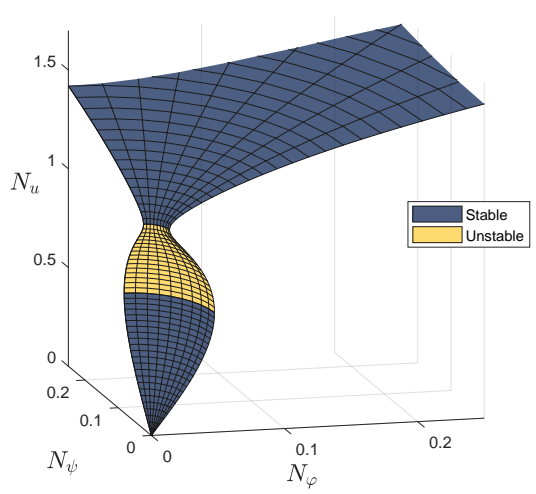

(b) $\delta=\frac{\pi}{2}$

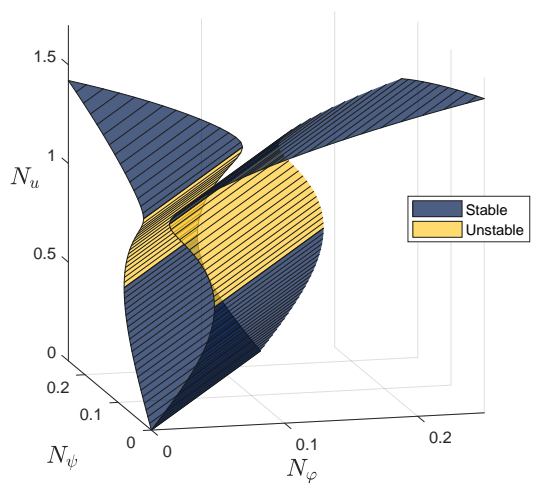

(d) $\delta=\pi$

Figure 4: The SIM of the system for several values of phase $\delta$ in the amplitude space $\left(N_{\varphi}, N_{\psi}, N_{u}\right)$. The unstable zone is reprensented in red.

\subsubsection{Stability of the SIM}

To determine the stability of each point of the SIM, we perturb system variables. In the Eq. 24, we introduce a small perturbation of $U$ as:

$$
U \rightarrow U+\Delta U, \Delta U \ll U
$$

After linearization, we obtain the following equation:

$$
\left[\begin{array}{c}
\frac{\partial(\Delta U)}{\partial \tau_{0}} \\
\frac{\partial\left(\Delta U^{\star}\right)}{\partial \tau_{0}}
\end{array}\right]=\underbrace{\left[\begin{array}{ll}
M_{1} & M_{2} \\
M_{2}^{\star} & M_{1}^{\star}
\end{array}\right]}_{\mathbf{M}}\left[\begin{array}{c}
\Delta U \\
\Delta U^{\star}
\end{array}\right]
$$

with

$$
M_{1}=-\frac{\lambda}{2}-\frac{i \omega}{2}+\frac{3 i \tilde{k}_{\mathrm{c}}}{4 \omega^{3}}|U|^{2} \quad \text { and } \quad M_{2}=\frac{3 i \tilde{k}_{\mathrm{c}}}{8 \omega^{3}} U^{2}
$$

and $*^{\star}$ stands for the complex conjugate of the considered function. The characteristic equation of the matrix $\mathbf{M}$ is:

$$
P_{\text {car }}(X)=X^{2}-\left(M_{1}+M_{1}^{\star}\right) X+\left|M_{1}\right|^{2}-\left|M_{2}\right|^{2}
$$


The sum and the product of the eigenvalues are given by $M_{1}+M_{1}^{\star}=-\lambda<0$ and $\left|M_{1}\right|^{2}-$ $\left|M_{2}\right|^{2}$ respectively. So the SIM is unstable if $\left|M_{1}\right|^{2}-\left|M_{2}\right|^{2}<0$, i.e.:

$$
\frac{27 \tilde{k}_{\mathrm{c}}^{2}}{64 \omega^{6}} N_{u}^{4}-\frac{3 \tilde{k}_{\mathrm{c}}}{4 \omega^{2}} N_{u}^{2}+\frac{\lambda^{2}}{4}+\frac{\omega^{2}}{4}<0
$$

which yield to:

$$
\frac{2 \omega^{3 / 2} \sqrt{2 \omega-\sqrt{\omega^{2}-3 \lambda^{2}}}}{3 \sqrt{\tilde{k}_{\mathrm{c}}}}<N_{u}<\frac{2 \omega^{3 / 2} \sqrt{2 \omega+\sqrt{\omega^{2}-3 \lambda^{2}}}}{3 \sqrt{\tilde{k}_{\mathrm{c}}}}
$$

The unstable zone is defined only by the value of $N_{u}$. It is represented in red on Fig. 4 .

\subsection{Slow time scale $\tau_{1}$, i.e. $\varepsilon^{1}$ order of system of equations}

Now, the system is described at the order $\varepsilon^{1}$. The first two equations of the system become:

$$
\left\{\begin{array}{l}
L g \frac{\partial \Phi}{\partial \tau_{1}}+\left(\frac{\omega^{2} c_{x}}{2}+i \frac{2 \sigma_{x} L g+a \omega\left(a \omega^{2}-g\right)}{2}\right) \Phi \\
\quad+i \cos (\alpha) \frac{\omega\left(a \omega^{2}-g\right)}{2} U+\frac{i \tilde{f}_{0} \cos (\beta) \omega^{2}}{2}=0 \\
\left(L g+k_{y}\right) \frac{\partial \Psi}{\partial \tau_{1}}+\left(\frac{\omega^{2} c_{y}}{2}+i \frac{2 \sigma_{y}\left(L g+k_{y}\right)+a \omega\left(a \omega^{2}-g\right)}{2}\right) \Psi \\
\quad+i \sin (\alpha) \frac{\omega\left(a \omega^{2}-g\right)}{2} U+\frac{i \tilde{f}_{0} \sin (\beta) \omega^{2}}{2}=0
\end{array}\right.
$$

or in a compact form, Eqs. 40 and 41 can be represented as:

$$
\frac{\partial \Phi}{\partial \tau_{1}}=f_{\varphi}(\Phi, \Psi, U) ; \quad \frac{\partial \Psi}{\partial \tau_{1}}=f_{\psi}(\Phi, \Psi, U)
$$

\subsubsection{Equilibrium points}

Equilibrium points of the system are obtained via considering the Eqs. 40 and 41 and evolution of the SIM at $\tau_{1}$ time scale [17]:

$$
\left\{\begin{array}{l}
f_{\varphi}(\Phi, \Psi, U)=0 \\
f_{\psi}(\Phi, \Psi, U)=0 \\
\left(\omega^{2}-i \omega \lambda\right) U+G(U)+\cos (\alpha)\left(a \omega^{2}-g\right) \Phi+\sin (\alpha)\left(a \omega^{2}-g\right) \Psi=0 \\
\operatorname{det}\left(\nabla_{\left(U, U^{\star}\right)} \mathcal{H}\right) \neq 0
\end{array}\right.
$$

where $\nabla_{\left(U, U^{\star}\right)} \mathcal{H}$ denotes the jacobian matrix of $\mathcal{H}$ versus variables $U$ and $U^{\star}$.

The Eqs. 43 and 44 give the expression of $\Phi$ and $\Psi$ as a function of $U$ :

$$
\left\{\begin{array}{l}
\Phi=-\frac{\cos (\alpha)\left(a \omega^{2}-g\right) U+\omega \tilde{f}_{0} \cos (\beta)}{2 L g \sigma_{x} / \omega+a^{2} \omega^{2}-a g-i c_{x} \omega} \\
\Psi=-\frac{\sin (\alpha)\left(a \omega^{2}-g\right) U+\omega \tilde{f}_{0} \sin (\beta)}{2\left(L g+k_{y}\right) \sigma_{y} / \omega+a^{2} \omega^{2}-a g-i c_{y} \omega}
\end{array}\right.
$$

By replacing the Eqs. 47 and 48 in the Eq. 45 and considering a cubic restoring force of the NES (see Eq. 23), one obtains:

$$
\left(\omega^{2}-i \omega \lambda\right) U-\frac{3 \tilde{k}_{\mathrm{c}}}{4 \omega^{2}} U|U|^{2}+(A+i B) U+C \tilde{f}_{0}=0
$$


where:

$$
\begin{gathered}
A=\operatorname{Re}\left(-\frac{\cos ^{2}(\alpha)\left(a \omega^{2}-g\right)^{2}}{2 L g \sigma_{x} / \omega+a^{2} \omega^{2}-a g-i c_{x} \omega}-\frac{\sin ^{2}(\alpha)\left(a \omega^{2}-g\right)^{2}}{2\left(L g+k_{y}\right) \sigma_{y} / \omega+a^{2} \omega^{2}-a g-i c_{y} \omega}\right) \\
B=\operatorname{Im}\left(-\frac{\cos ^{2}(\alpha)\left(a \omega^{2}-g\right)^{2}}{2 L g \sigma_{x} / \omega+a^{2} \omega^{2}-a g-i c_{x} \omega}-\frac{\sin ^{2}(\alpha)\left(a \omega^{2}-g\right)^{2}}{2\left(L g+k_{y}\right) \sigma_{y} / \omega+a^{2} \omega^{2}-a g-i c_{y} \omega}\right) \\
C=-\frac{\cos (\alpha)\left(a \omega^{2}-g\right) \omega \cos (\beta)}{2 L g \sigma_{x} / \omega+a^{2} \omega^{2}-a g-i c_{x} \omega}-\frac{\sin (\alpha)\left(a \omega^{2}-g\right) \omega \sin (\beta)}{2\left(L g+k_{y}\right) \sigma_{y} / \omega+a^{2} \omega^{2}-a g-i c_{y} \omega}
\end{gathered}
$$

The complex variable $U$ is expressed in the polar form as $U=N_{u} e^{i \delta_{u}}$. Eq. 49 reads:

$$
\left[-\frac{3 \tilde{k}_{\mathrm{c}}}{4 \omega^{2}} N_{u}^{3}+\left(\omega^{2}+A+i(B-\omega \lambda)\right) N_{u}\right] e^{i \delta_{u}}=-C \tilde{f}_{0}
$$

The modules of both sides of the Eq. 53 are equal:

$$
\begin{gathered}
\left(-\frac{3 \tilde{k}_{\mathrm{c}}}{4 \omega^{2}} N_{u}^{3}+\left(\omega^{2}+A\right) N_{u}\right)^{2}+(B-\omega \lambda)^{2} N_{u}^{2}-|C|^{2} \tilde{f}_{0}^{2}=0 \\
\frac{9 \tilde{k}_{\mathrm{c}}^{2}}{16 \omega^{4}} N_{u}^{6}-\frac{3 \tilde{k}_{\mathrm{c}}}{2 \omega^{2}}\left(\omega^{2}+A\right) N_{u}^{4}+\left[\left(\omega^{2}+A\right)^{2}+(B-\omega \lambda)^{2}\right] N_{u}^{2}-|C|^{2} \tilde{f}_{0}^{2}=0
\end{gathered}
$$

The Eq. 55 is a polynomial equation of degree three in $N_{u}^{2}$. It is possible to solve it with Cardano's method. Then, the phase angle $\delta_{u}$ is calculated thanks to the Eq. 53 as:

$$
\delta_{u}=\arg \left(\frac{-C \tilde{f}_{0}}{-\frac{3 \tilde{k}_{\mathrm{c}}}{4 \omega^{2}} N_{u}^{3}+\left(\omega^{2}+A+i(B-\omega \lambda)\right) N_{u}}\right)
$$

The Eq. 25 describing the SIM can be separated in two complex conjugate equations as:

$$
\begin{aligned}
& \frac{\lambda+i \omega}{2} U-i \frac{3 \tilde{k}_{\mathrm{c}}}{8 \omega^{3}} U^{2} U^{\star}+i \cos (\alpha) \frac{a \omega^{2}-g}{2 \omega} \Phi+i \sin (\alpha) \frac{a \omega^{2}-g}{2 \omega} \Psi=0 \\
& \frac{\lambda-i \omega}{2} U^{\star}+i \frac{3 \tilde{k}_{\mathrm{c}}}{8 \omega^{3}} U U^{\star 2}-i \cos (\alpha) \frac{a \omega^{2}-g}{2 \omega} \Phi^{\star}-i \sin (\alpha) \frac{a \omega^{2}-g}{2 \omega} \Psi^{\star}=0
\end{aligned}
$$

Then, to determine if the point is an equilibrium point or a singular point, one should evaluate the determinant $\operatorname{det}\left(\nabla_{\left(U, U^{\star}\right)} \mathcal{H}\right)$ :

$$
\nabla_{\left(U, U^{\star}\right)} \mathcal{H}=\left[\begin{array}{cc}
\frac{\partial \mathcal{H}}{\partial U} & \frac{\partial \mathcal{H}}{\partial U^{\star}} \\
\frac{\partial \mathcal{H}^{\star}}{\partial U} & \frac{\partial \mathcal{H}^{\star}}{\partial U^{\star}}
\end{array}\right]=\left[\begin{array}{cc}
\frac{\lambda+i \omega}{2}-i \frac{3 \tilde{k}_{\mathrm{c}}}{4 \omega^{3}} U U^{\star} & -i \frac{3 \tilde{k}_{\mathrm{c}}}{8 \omega^{3}} U^{2} \\
i \frac{3 \tilde{k}_{\mathrm{c}}}{8 \omega^{3}} U^{\star 2} & \frac{\lambda-i \omega}{2}+i \frac{3 \tilde{k}_{\mathrm{c}}}{4 \omega^{3}} U U^{\star}
\end{array}\right]=-\mathbf{M}
$$

The matrix $\mathbf{M}$ has been already seen in Eq. 35 for tracing stability zones of the SIM. We can conclude that the point is singular if it is located on the border of the unstable zone of the SIM, copying as:

$$
N_{u}=\frac{2 \omega^{3 / 2} \sqrt{2 \omega-\sqrt{\omega^{2}-3 \lambda^{2}}}}{3 \sqrt{\tilde{k}_{\mathrm{c}}}} \quad \text { and } \quad N_{u}=\frac{2 \omega^{3 / 2} \sqrt{2 \omega+\sqrt{\omega^{2}-3 \lambda^{2}}}}{3 \sqrt{\tilde{k}_{\mathrm{c}}}}
$$

Figure 5 summarizes detected equilibrium points in terms of $N_{u}$ as a function of the frequency of excitation $\Omega$ for different values of $f_{0}$. It is seen that, there is possibility of multiple equilibrium points according to $\Omega$ varying from one to three solutions. Moreover, for some forcing amplitudes, isolated branches exist. These isolated branches should be investigated very well as they can correspond to high energy levels for the main system. 


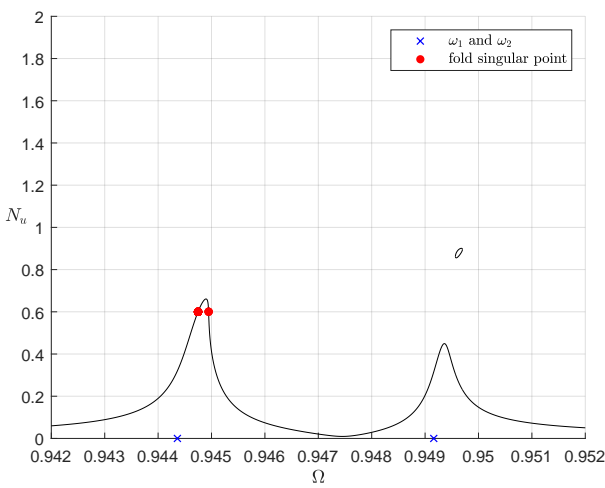

(a) $f_{0}=5 \varepsilon$

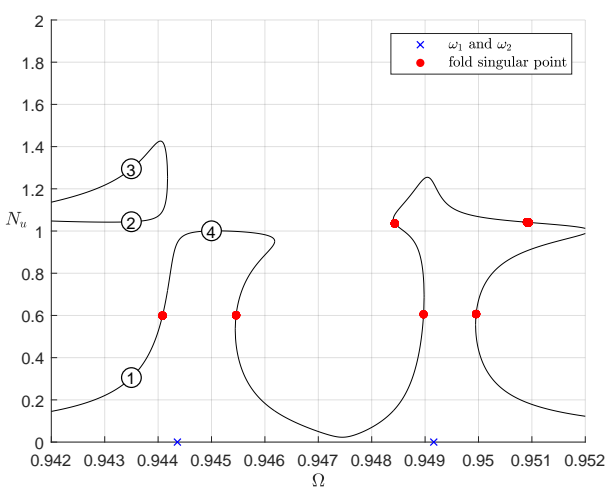

(c) $f_{0}=12 \varepsilon$

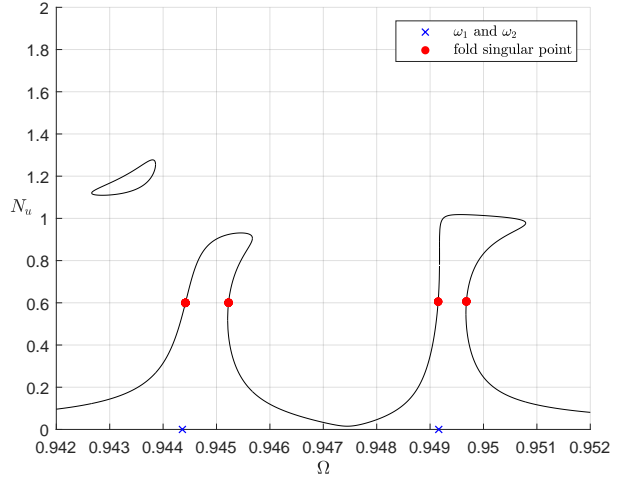

(b) $f_{0}=8 \varepsilon$

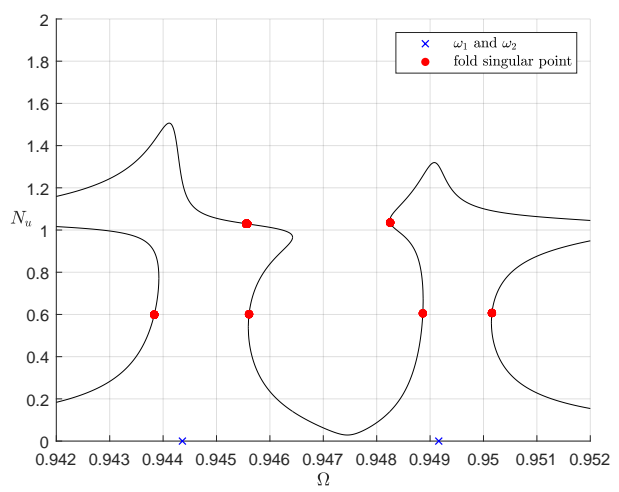

(d) $f_{0}=15 \varepsilon$

Figure 5: Values of $N_{u}$ for equilibrium points as a function of $\Omega$ with several values of $f_{0}$. Points $(\bullet)$ correspond to fold singular points. Crosses $(\times)$ correspond to the natural frequencies of the main system $\omega_{1}$ and $\omega_{2}$.

\subsubsection{Stability of equilibrium points}

The equilibrium points that are located in the unstable zone of the SIM are always unstable. The equilibrium points out of this zone are not necessarily stable. To determine their stability, we can plot the phase portraits around them. Evolution of the SIM (Eq. 25) at $\tau_{1}$ time scale leads to:

$$
\nabla_{\left(\delta_{u}, N_{u}, \delta_{\varphi}, N_{\varphi}, \delta_{\psi}, N_{\psi}\right)} \mathcal{H}\left(\begin{array}{lllll}
\frac{\partial \delta_{u}}{\partial \tau_{1}} & \frac{\partial N_{u}}{\partial \tau_{1}} & \frac{\partial \delta_{\varphi}}{\partial \tau_{1}} & \frac{\partial N_{\varphi}}{\partial \tau_{1}} & \frac{\partial \delta_{\psi}}{\partial \tau_{1}}
\end{array} \quad \frac{\partial N_{\psi}}{\partial \tau_{1}}\right)^{\mathrm{T}}=0
$$

or

$$
\left(\begin{array}{c}
\frac{\partial \delta_{u}}{\partial \tau_{1}} \\
\frac{\partial N_{u}}{\partial \tau_{1}}
\end{array}\right)=\left(\nabla_{\left(\delta_{u}, N_{u}\right)} \mathcal{H}\right)^{-1} \nabla_{\left(\delta_{\varphi}, N_{\varphi}, \delta_{\psi}, N_{\psi}\right)} \mathcal{H} \underbrace{\left(\begin{array}{lll}
\frac{\partial \delta_{\varphi}}{\partial \tau_{1}} & \frac{\partial N_{\varphi}}{\partial \tau_{1}} \frac{\partial \delta_{\psi}}{\partial \tau_{1}} & \frac{\partial N_{\psi}}{\partial \tau_{1}}
\end{array}\right)^{\mathrm{T}}}_{\mathcal{L}}
$$

The values of $\delta_{\varphi}, N_{\varphi}, \delta_{\psi}$ and $N_{\psi}$ are the solution of each equilibrium point. Considering the Eqs. 40 and 41 , the vector $\mathcal{L}$ can be evaluated easily. To plot the phase portraits, the values of $N_{u}$ and $\delta_{u}$ vary around the equilibrium point.

Fig. 6 shows the phase portraits of four equilibrium points labeled on Fig. 5c.

According to the phase portraits, the points (1), (2) and (3) are stable. The point (4) 


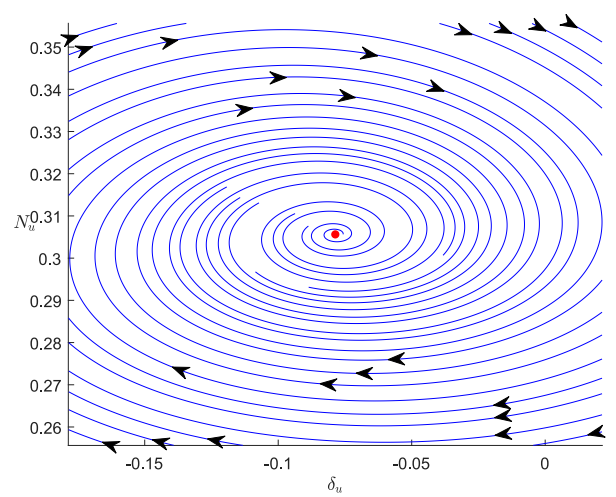

(a) Point (1)

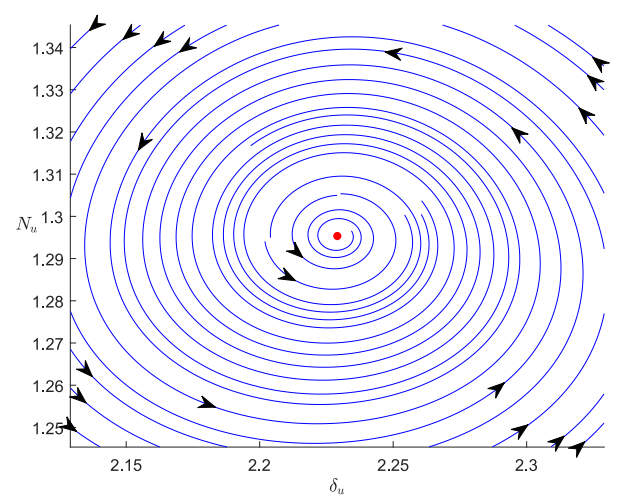

(c) Point (3)

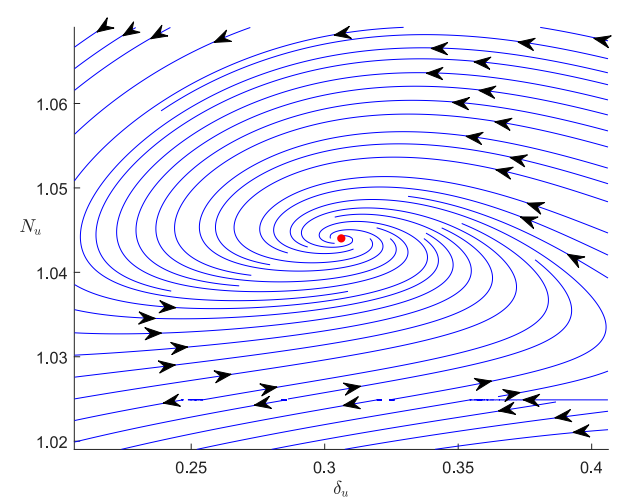

(b) Point (2)

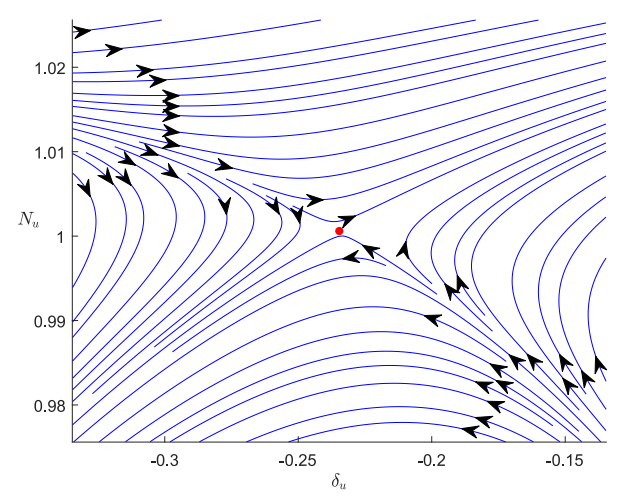

(d) Point (4)

Figure 6: Phase portraits around four equilibrium points of the Fig. 5c.

is located in the unstable zone of the SIM, so it is unstable. Apart from that, its phase portrait (see Fig. 6d) shows a saddle point.

\section{Numerical results}

In this section, the analytical results are compared to direct numerical time integration of Eqs. 8, 9 and 10. The aim is to validate the assumption we made for the analytic developments and to determine the influence of following parameters: $\varepsilon, \alpha, f_{0}$ and $\Omega$. Direct integration of Eqs. 8, 9 and 10 are carried out by the ode 45 function of MATLAB ${ }^{\circledR}$ with relative tolerance at $10^{-14}$ and absolute tolerance at $10^{-14}$.

\subsection{Free oscillations}

\subsubsection{Influence of $\varepsilon$}

Let us consider that the system is under only initial condition, i.e. $f_{0}=0$. We set following initial conditions:

$$
(\tilde{\varphi}(0), \tilde{\psi}(0), \tilde{u}(0), \dot{\tilde{\varphi}}(0), \dot{\tilde{\psi}}(0), \dot{\tilde{u}}(0))=\left(0.2,0.1, \tilde{u}_{0}, 0,0,0\right)
$$


The value of $\tilde{u}_{0}$ is calculated in the static equilibrium as:

$$
\tilde{u}_{0}=\left(-\frac{\varepsilon g}{\tilde{k}_{\mathrm{c}}}[\sin (\alpha) \sin (\tilde{\psi}(0))+\cos (\alpha) \cos (\tilde{\psi}(0)) \sin (\tilde{\varphi}(0))]\right)^{\frac{1}{3}}
$$

In the analytical developments, we assumed that $\varepsilon$ is very small. The Fig. 7 shows the path of the system in the amplitude space $\left(N_{\varphi}, N_{\psi}, N_{u}\right)$ with the SIM for several values of $\varepsilon$. Note that the SIM is plotted for $\delta=\frac{\pi}{2}$ but in reality its shape is evolving with respect to $\delta$. From Fig. 7, we note that for high value of $\varepsilon$, the amplitude $N_{u}$ is not converging to zero.

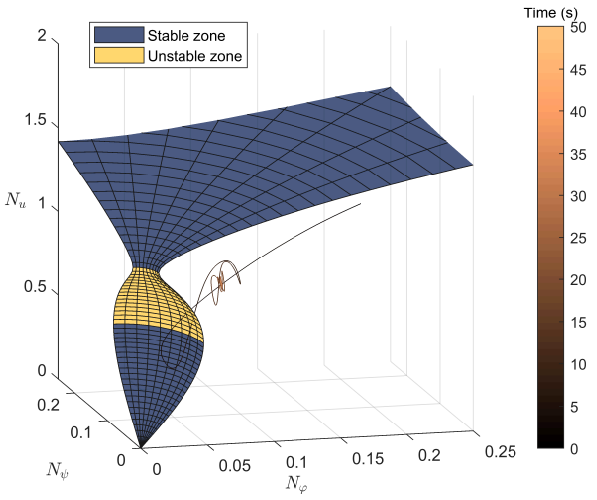

(a) $\varepsilon=10^{-1}$

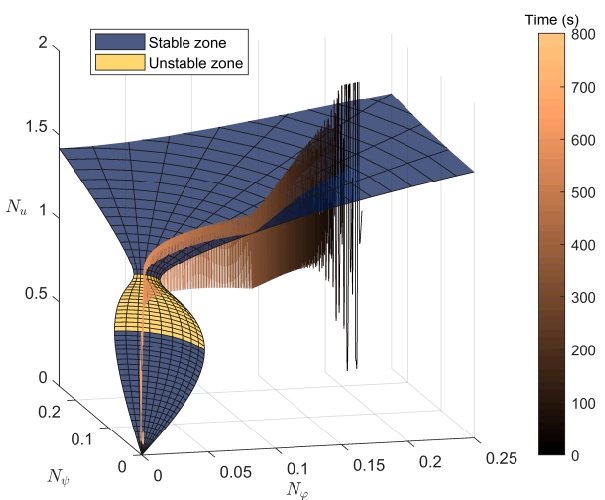

(c) $\varepsilon=10^{-3}$

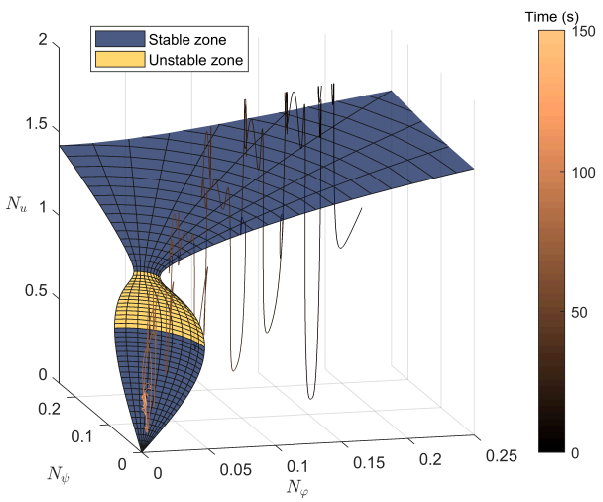

(b) $\varepsilon=10^{-2}$

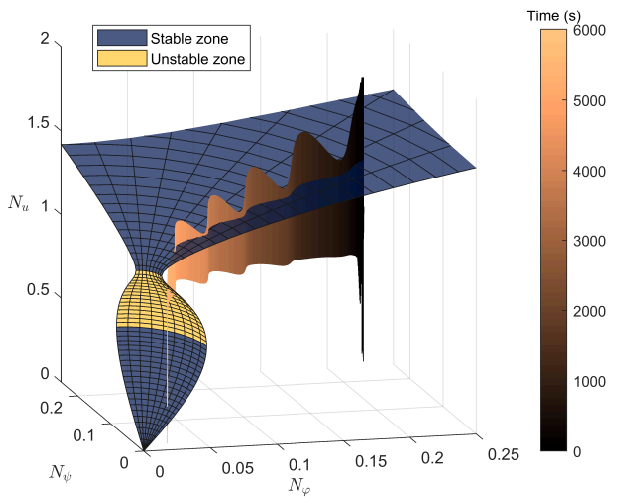

(d) $\varepsilon=10^{-4}$

Figure 7: The SIM for $\delta=\frac{\pi}{2}$ and corresponding numerical results for several values of $\varepsilon$ in space of amplitude. Numerical results are obtained via direct integration of Eqs. 8, 9 and 10 for the system with $f_{0}=0$ and under initial conditions as descirbed in Eq. 61.

In fact, there are three static equilibria of the system. These points can be obtained from Eq. 8,9 and 10 via setting $\ddot{\varphi}=\ddot{\psi}=\ddot{u}=\dot{\varphi}=\dot{\psi}=\dot{u}=0$. The state $\varphi=\psi=u=0$ is one of them but it is unstable.

It is seen that as $\varepsilon$ decreases, the numerical results follow the SIM. With small values of $\varepsilon$, the amplitudes decreases slowly along the SIM to reach to the border of the unstable zone. Here, the value of $N_{u}$ jumps to low value whereas the amplitudes $N_{\varphi}$ and $N_{\psi}$ continue to vary slowly. For the same initial conditions, the time to reach the stability border and then to bifurcate is highly dependent of the parameter $\varepsilon$.

In the rest of the section, we take $\varepsilon=10^{-4}$ in order to obtain the most readable results. 


\subsection{Forced system}

Let us assume that the system is under a generalized external excitation with amplitude $f_{0}$ and the frequency $\Omega$. The initial conditions are set to be zero, i.e.:

$$
(\tilde{\varphi}(0), \tilde{\psi}(0), \tilde{u}(0), \dot{\tilde{\varphi}}(0), \dot{\tilde{\psi}}(0), \dot{\tilde{u}}(0))=(0,0,0,0,0,0)
$$

\subsubsection{Influence of $\alpha$}

The distinctive characteristic of the considered system is using a single NES to control two degrees of freedom of the pendulum. This is possible thanks to the inclined positioning of the NES with the angle $\alpha$. The choice of this angle is important for the behavior of the system. In particular, the analytical study shows the NES can be decoupled to the main system for values of the phase $\delta=k \pi$ where $k$ is an odd number and $\frac{N_{\varphi}}{N_{\psi}}=\tan (\alpha)$. As already explained, this case occurs when the pendulum oscillates in the orthogonal position with respect to the direction of the NES.

Let us consider some cases to clarify the significance of this issue. In the following four cases, the forcing angle $\beta=\alpha+\frac{\pi}{2}$ (see Fig. 3):

(a) $\alpha=0, \beta=\frac{\pi}{2}, \omega_{1} \neq \omega_{2}$;

(b) $\alpha=\frac{\pi}{4}, \beta=\frac{3 \pi}{4}, \omega_{1} \neq \omega_{2}$;

(c) $\alpha=\frac{\pi}{4}, \beta=\frac{3 \pi}{4}, \omega_{1}=\omega_{2}, c_{x}=c_{y}$;

(d) $\alpha=\frac{\pi}{4}, \beta=\frac{3 \pi}{4}, \omega_{1}=\omega_{2}, c_{x} \neq c_{y}$.

For discussing about all four above mentioned items, we consider following characteristics for the external excitation: $f_{0}=10$ and $\Omega=\omega_{2}$. Numerical results presented on Fig. 8 show that the NES is not activated on case (a) and (c).

- Case (a): In the absence of any internal or autoparemetric resonances, we can claim that since the excitation acts only in the $\vec{e}_{y}$ direction, only the oscillations around $\vec{e}_{x}(\psi)$ is concerned. In this case, the NES is in the $\vec{e}_{x}$ direction so it is not activated.

- Case (b): Even in the absence of any internal or autoparemetric resonances, both oscillations around $\vec{e}_{y}(\varphi)$ and $\vec{e}_{x}(\psi)$ are activated and the NES affects the overall behavior of the system.

- Case (c): The Eqs. 47 and 48 become:

$$
\Phi=-\frac{\cos (\alpha)\left(a \omega^{2}-g\right) U-\omega \tilde{f}_{0} \sin (\alpha)}{2 L g \sigma_{x} / \omega+a^{2} \omega^{2}-a g-i c_{x} \omega} ; \quad \Psi=-\frac{\sin (\alpha)\left(a \omega^{2}-g\right) U+\omega \tilde{f}_{0} \cos (\alpha)}{2 L g \sigma_{x} / \omega+a^{2} \omega^{2}-a g-i c_{x} \omega}
$$

By replacing in the Eq. 45 and considering a cubic restoring force of the NES, we can write:

$$
\underbrace{\left(\omega^{2}-i \omega \lambda-\frac{3 \tilde{k}_{\mathrm{c}}}{4 \omega^{2}}|U|^{2}+A+i B\right)}_{\mathcal{R}} U=0
$$

The imaginary part of the first member of the Eq. 65 reads:

$$
\operatorname{Im}(\mathcal{R})=-\frac{\left(a \omega^{2}-g\right)^{2} c_{x} \omega}{\left(2 L g \sigma_{x} / \omega+a^{2} \omega^{2}-a g\right)^{2}+\left(c_{x} \omega\right)^{2}}-\omega \lambda<0
$$

Since the expression $\mathcal{R}$ is nonzero, the only solution of the Eq. 65 is $U=0$. In this case the NES is not activated. 


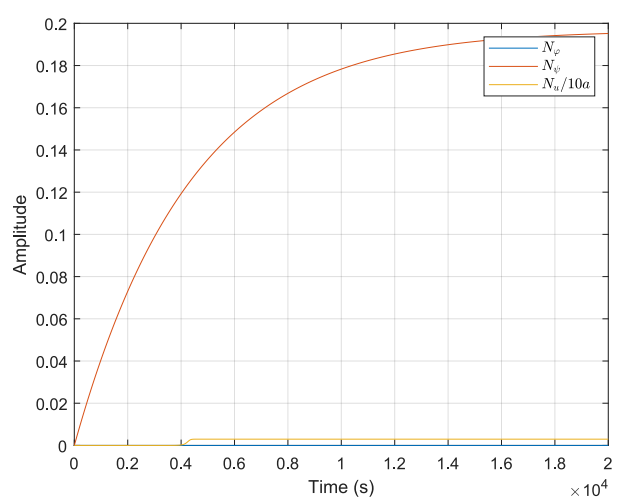

(a) : $\alpha=0, \beta=\frac{\pi}{2}, \omega_{1} \neq \omega_{2}$

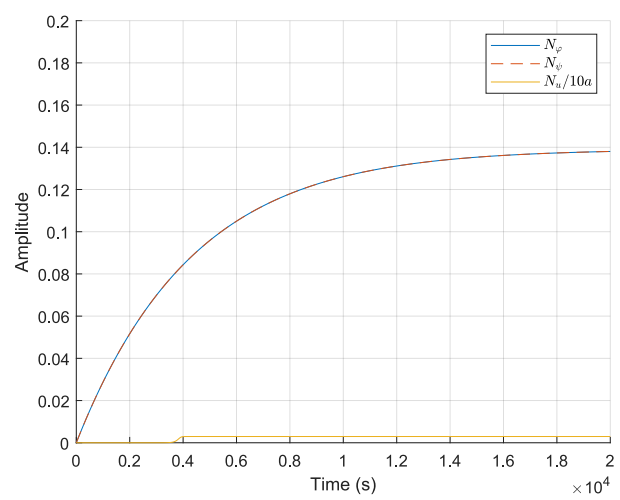

(c) : $\alpha=\frac{\pi}{4}, \beta=\frac{3 \pi}{4}, \omega_{1}=\omega_{2}, c_{y}=c_{x}$

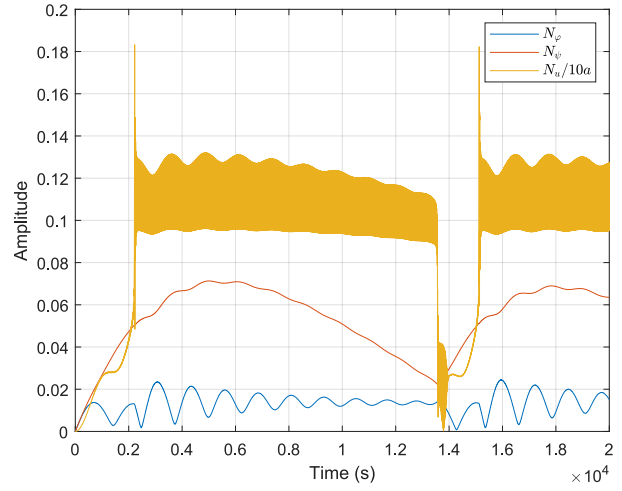

(b) : $\alpha=\frac{\pi}{4}, \beta=\frac{3 \pi}{4}, \omega_{1} \neq \omega_{2}$

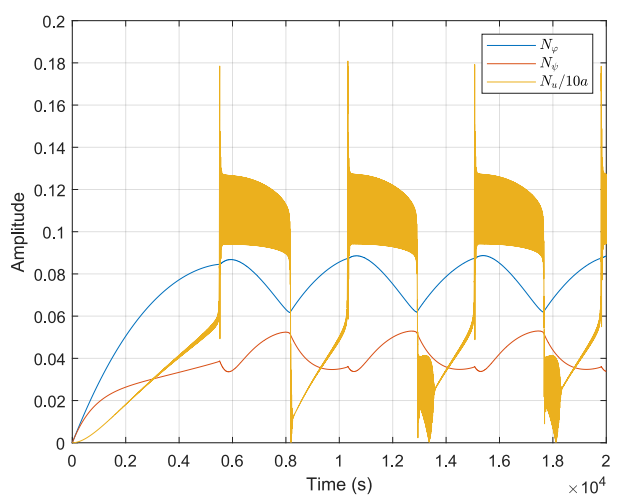

(d) $: \alpha=\frac{\pi}{4}, \beta=\frac{3 \pi}{4}, \omega_{1}=\omega_{2}, c_{y} \neq c_{x}$

Figure 8: Four cases of numerical free oscillations with forcing angle $\beta$ orthogonal to the direction of the NES.

Considering $U=0$ and $\alpha=\frac{\pi}{4}$, the Eqs. 64 lead to $\Psi=-\Phi$. So $\delta=\pi$ which means that the SIM is degenerate (see Fig. $4 \mathrm{~d}$ ).

- Case (d): this a general case where $\Psi \neq-\Phi$. The phase $\delta$ is not equal to $\pi$, thus the SIM is not always degenerate and the NES is activated.

As a summary, the necessary condition for activation of the NES is that $\alpha \neq k \frac{\pi}{2}, k \in \mathbb{Z}$. This condition is not enough as if $\omega_{1}=\omega_{2}$ and $c_{x}=c_{y}$, the NES can be not activated.

\subsubsection{Influence of the amplitude of excitation}

In all the simulations the angle of the NES is set as $\alpha=\frac{\pi}{6}$ and the angle of the excitation as $\beta=\frac{\pi}{4}$.

In the first example, the amplitude of the force is $f_{0}=5 \varepsilon$ and the frequency $\Omega=$ $0.945 \mathrm{rads}^{-1}$. As shown in Fig. 9, the system goes to a stable equilibrium point. In section 3.2.2, we have seen that several stable equilibrium points can exist for one condition of excitation. Fig. 10 shows a numerical integration with the condition of the points (1), (2), (3) labeled on the Fig. 5c. The results are plotted in the amplitude space $\left(N_{\varphi}, N_{\psi}, N_{u}\right)$ with the SIM and on the phase portrait. The three different equilibrium points are labeled with the same numbers (1), (2) and (3). The system is attracting by the equilibrium point (3) 


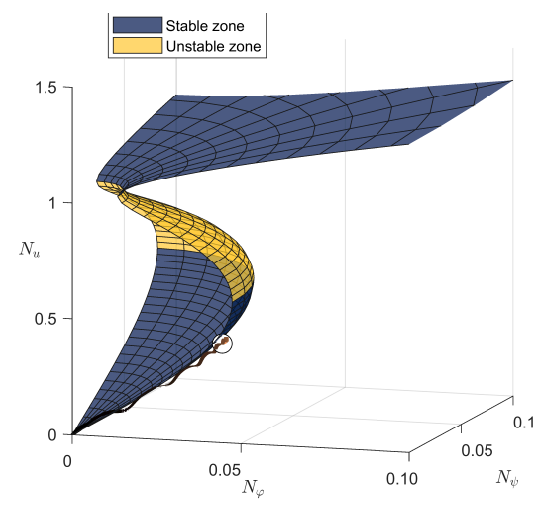

(a) Amplitude space

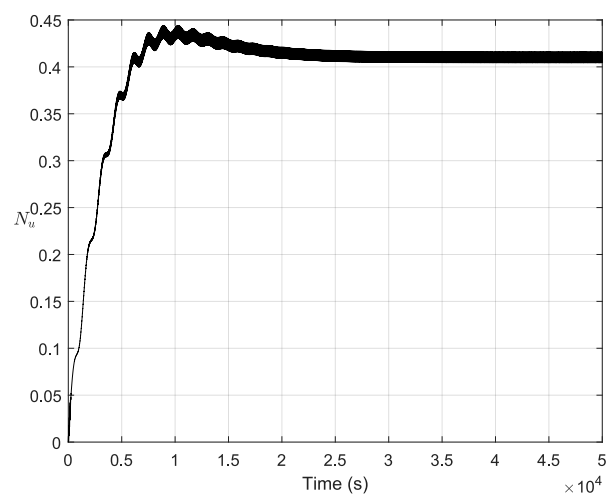

(c) NES amplitude $N_{u}$

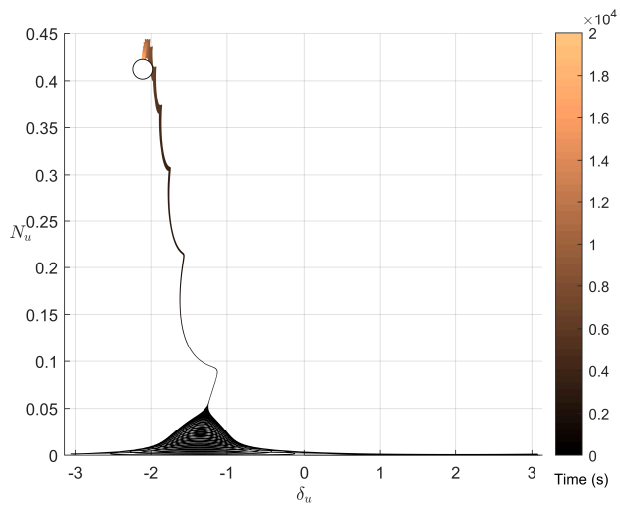

(b) Phase portrait

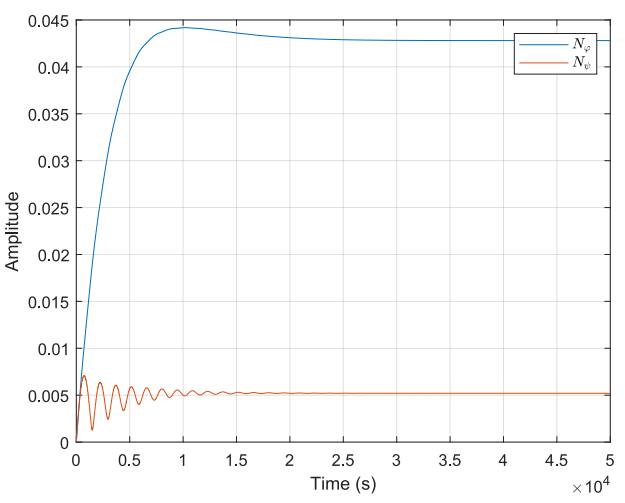

(d) Pendulum angles amplitude

Figure 9: Numerical integration with $f_{0}=5 \varepsilon, \Omega=0.945 \mathrm{rad} \mathrm{s}^{-1}$.

located on an isolated branch. In the last example illustrated in Fig. 11, the conditions are those of the equilibrium point (4) labeled on the Fig. 5c. In this case, only one equilibrium point exists but it is unstable. Consequently, the system is oscillating around the unstable zone of the SIM. These oscillations are called SMR oscillations [18]. They are used to limit the amplitude of the main system below a threshold. 


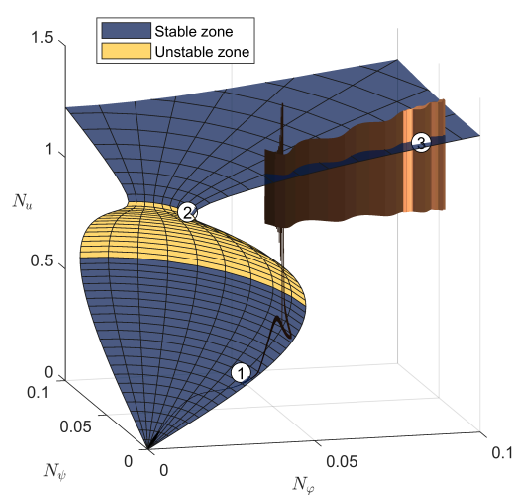

(a) Amplitude space

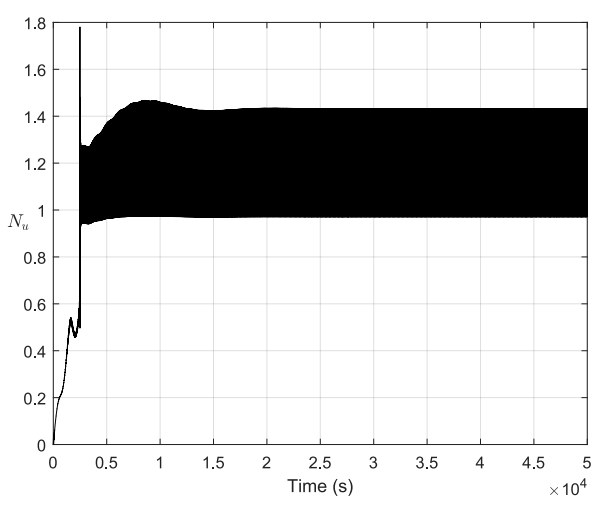

(c) NES amplitude $N_{u}$

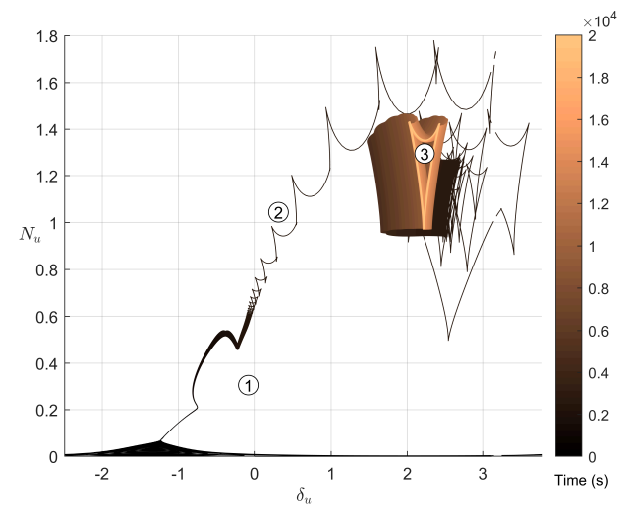

(b) Phase portrait

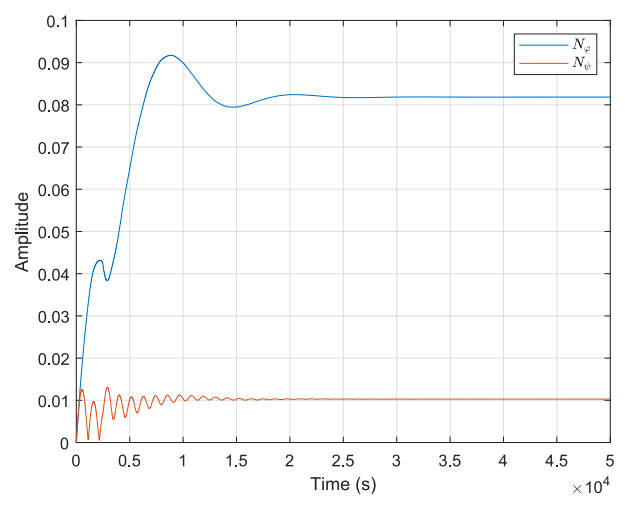

(d) Pendulum angles amplitude

Figure 10: Numerical integration with $f_{0}=12 \varepsilon, \Omega=0.9435 \mathrm{rad} \mathrm{s}^{-1}$. 


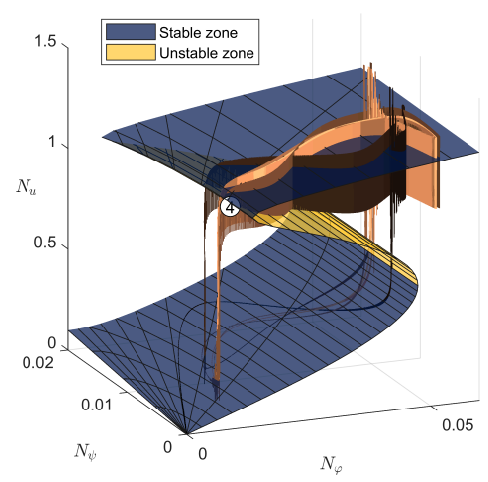

(a) Amplitude space

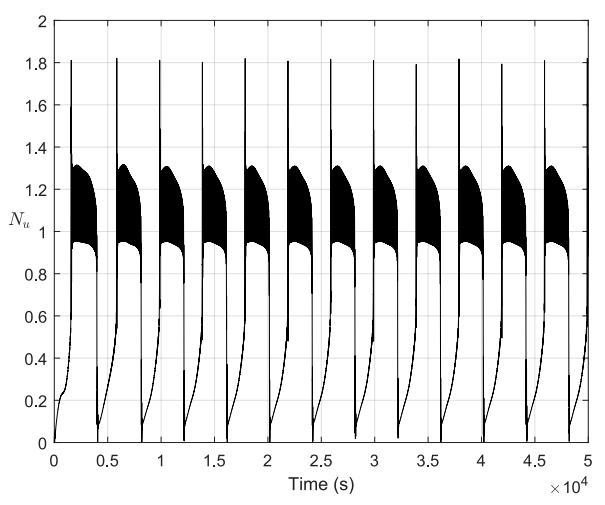

(c) NES amplitude $N_{u}$

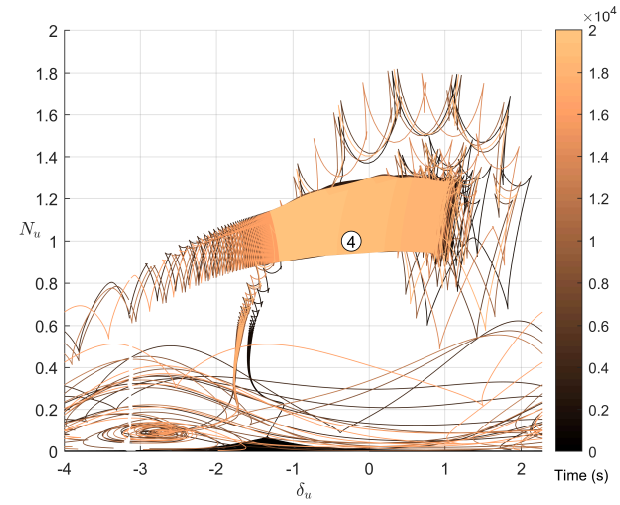

(b) Phase portrait

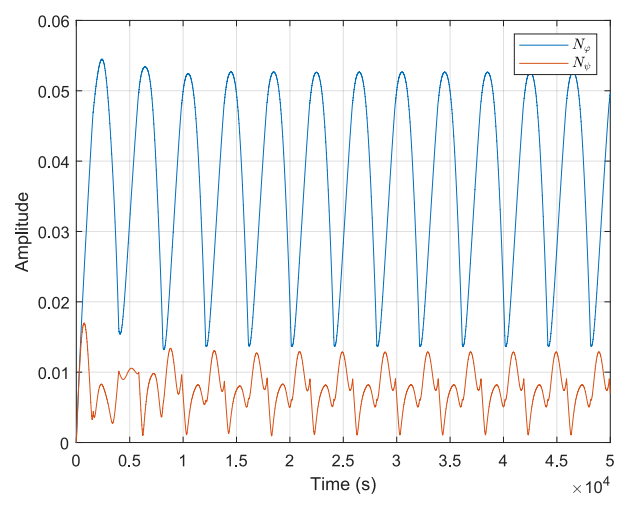

(d) Pendulum angles amplitude

Figure 11: Numerical integration with $f_{0}=12 \varepsilon, \Omega=0.945 \mathrm{rad} \mathrm{s}^{-1}$. 


\section{Non-smooth nonlinear energy sink}

For the sake of application, a NES with non-polynomial nonlinearity can be considered [10]. The passive control of a main oscillator by a nonsmooth NES is studied by Lamarque et al. [19] while the same phenomenon in gravitational field is analyzed by Ture Savadkoohi et al. [20]. In this section, a brief summary of results for our system under consideration coupled with a non-smooth NES are presented. Let us assume following restoring forcing function for the NES:

$$
S(u)= \begin{cases}K_{l}(u+d) & \text { if } u<-d \\ 0 & \text { if }-d \leq u \leq d \\ K_{l}(u-d) & \text { if } u>d\end{cases}
$$

where $K_{l}$ and $d$ are parameters and $u$ is the relative displacement of the mass $m$ in the local reference frame. The rescaling of the system equations provides a new definition of the force as $s(u)=\frac{S(u)}{M}$ :

$$
s(u)= \begin{cases}\varepsilon k_{l}(\tilde{u}+\tilde{d}) & \text { if } \tilde{u}<-\tilde{d} \\ 0 & \text { if }-\tilde{d} \leq \tilde{u} \leq \tilde{d} \\ \varepsilon k_{l}(\tilde{u}-\tilde{d}) & \text { if } \tilde{u}>\tilde{d}\end{cases}
$$

where $\tilde{d}=\frac{d}{\varepsilon}$ and $k_{l}=\frac{K_{l}}{m}$.

The expression of the function $G(U)$ (see Eq. 23 for cubic NES) reads [21]:

$$
G(U)=\frac{\Omega}{2 \pi} \int_{0}^{\frac{2 \pi}{\Omega}} s\left(\frac{U e^{i \Omega t}-U^{\star} e^{-i \Omega t}}{2 i \Omega}\right) e^{-i \Omega \tau_{0}} \mathrm{~d} \tau_{0}=-\frac{i \tilde{k}_{l} U}{2 \omega} H(|U|)
$$

with

$$
H(|U|)= \begin{cases}0 & \text { if }|U|<D \\ \frac{1}{\pi}\left(2 \arccos \left(\frac{D}{|U|}\right)-\frac{2 D \sqrt{|U|^{2}-D^{2}}}{|U|^{2}}\right) & \text { if }|U| \geq D\end{cases}
$$

where $D=\omega \tilde{d}$. The SIM is shown on Fig. 12. For values of $N_{u}<D$, the behavior of the system is linear. If the amplitude of $u$ increases, the behavior becomes nonlinear. There are an unstable zone colored in red and a stable zone. The singular points are located on the border of the unstable zone.

The amplitude-frequency curves of the system $\left(N_{u}\right.$ versus $\left.\Omega\right)$ under different external forcing terms are provided in Fig. 13. As for cubic force, there may be one or three solutions for the same parameters. There are also isolated branches. An example of SMR oscillations is given on Fig. 14. 


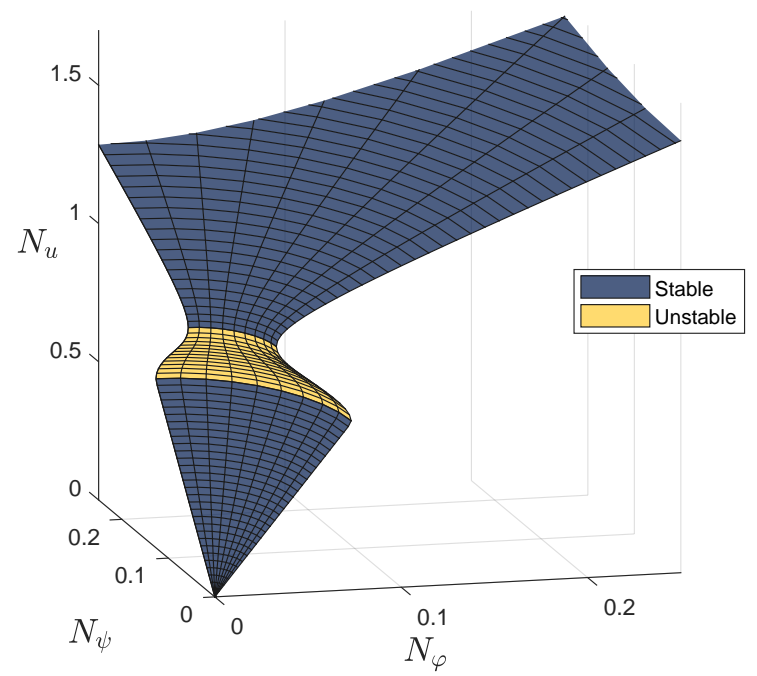

Figure 12: The SIM of the system for $\delta=\frac{\pi}{2}, \tilde{d}=0.6 \mathrm{~m}$ and $k_{l}=4 \mathrm{~s}^{-2}$ in the amplitude space $\left(N_{\varphi}, N_{\psi}, N_{u}\right)$. The unstable zone is reprensented in red.

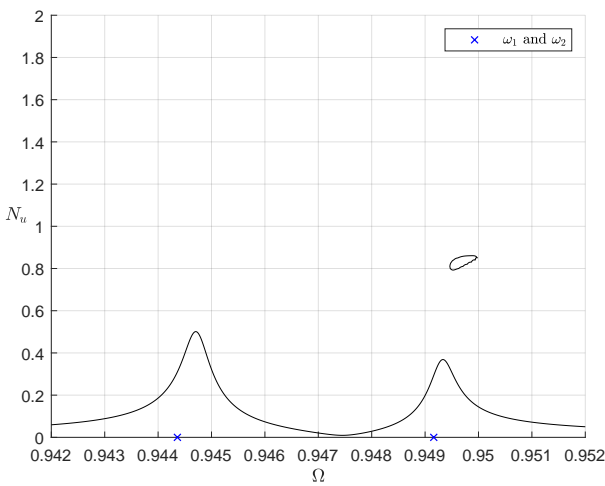

(a) $f_{0}=5 \varepsilon$

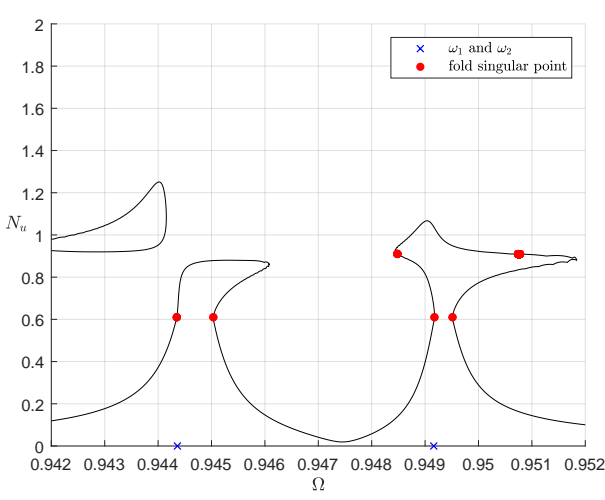

(c) $f_{0}=10 \varepsilon$

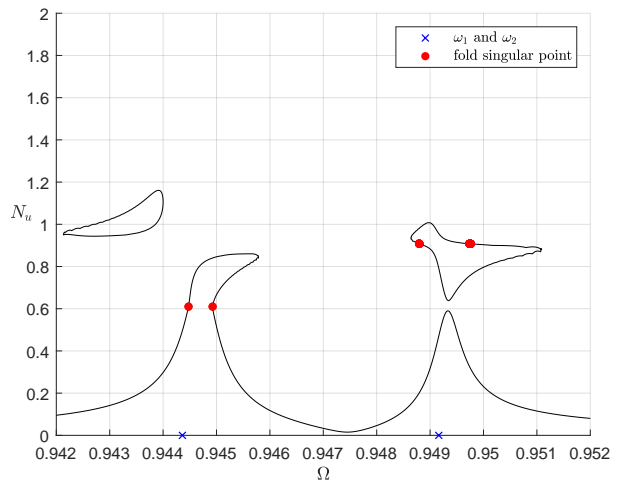

(b) $f_{0}=8 \varepsilon$

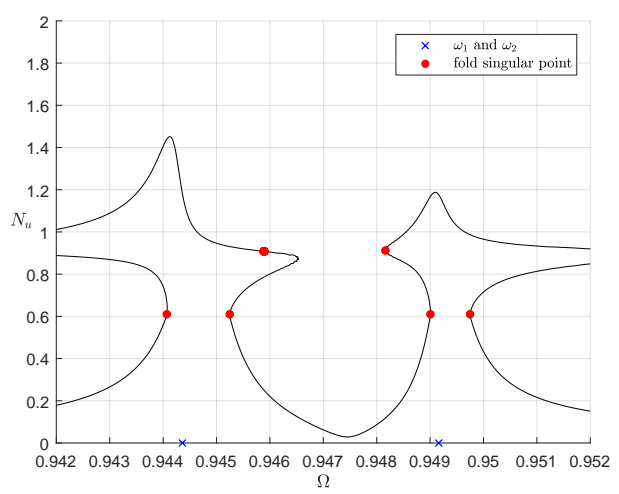

(d) $f_{0}=15 \varepsilon$

Figure 13: Values of $N_{u}$ for equilibrium points as a function of $\Omega$ with several values of $f_{0}$. Points $(\bullet)$ correspond to fold singular points. Crosses $(\times)$ correspond to the natural frequencies of the main system $\omega_{1}$ and $\omega_{2}$. 


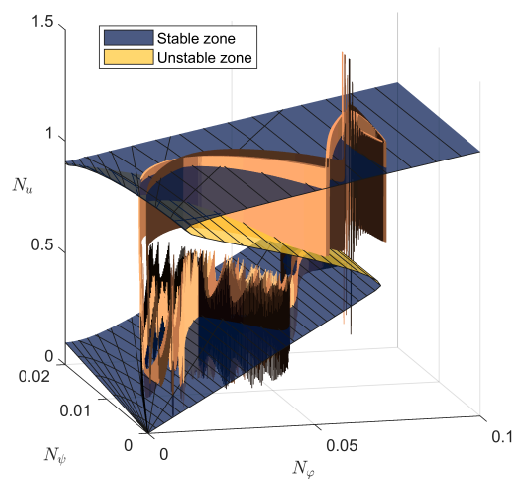

(a) Amplitude space

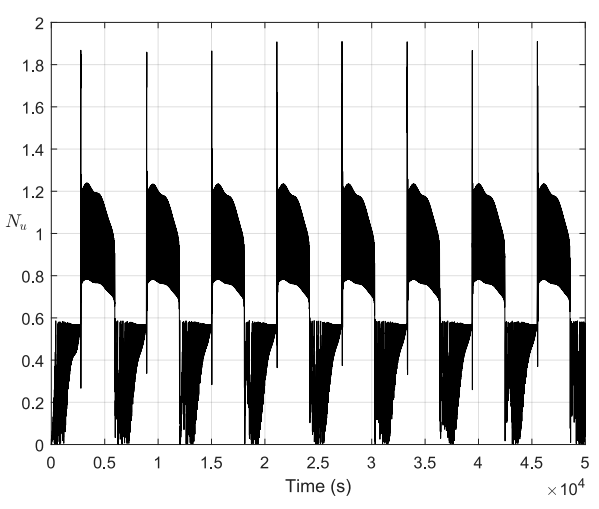

(c) NES amplitude $N_{u}$

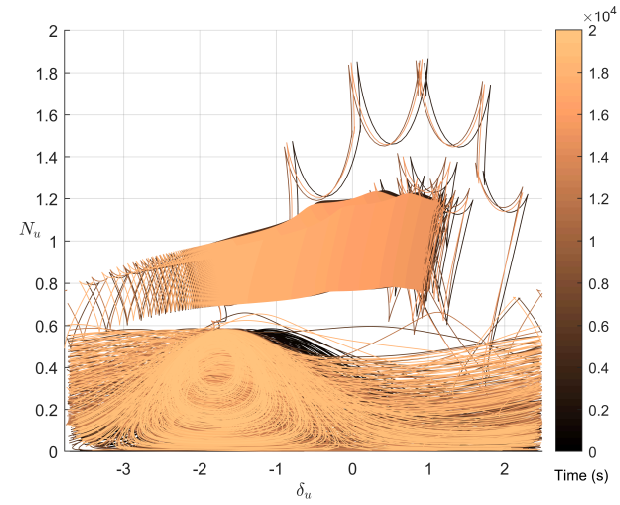

(b) Phase portrait

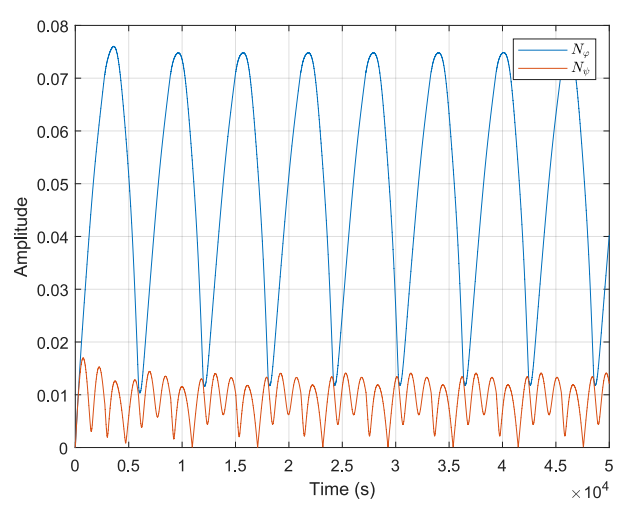

(d) Pendulum angles amplitude

Figure 14: Numerical integration with $f_{0}=12 \varepsilon, \Omega=0.945 \mathrm{rad} \mathrm{s}^{-1}$. 


\section{Conclusion}

The passive control of a two-degrees-of-freedom pendulum by a nonlinear absorber, which is positioned in an arbitrary direction with respect to both directions of oscillations, is studied. Due to practical applications, oscillations of the pendulum in one of the directions is subjected to a semi-rigid constraint by introducing a rotational rigidity at its base. The complexified form of system equations are treated via a multiple scale method leading to detection of its slow invariant manifold and equilibrium and singular points. Detected stability zones of the phase-dependent slow invariant manifold could explain the possible bifurcation of the system during a free vibration (depending on initial conditions), while traced singularities and equilibrium points for the forced system enlightened the reason for modulated responses and periodic regimes, respectively. Further studies on system singularities and equilibrium points lead to tracing amplitude-frequency curves showing possibility of existence of isolated branches, which could correspond to high energy levels for pendulum oscillations. It is also demonstrated that the orientation angle of the nonlinear absorber with respect to the direction of oscillations is important as it is possible that the absorber cannot be activated. The design process of the nonlinear absorber should be carried out in a way that these characteristic points of the system under a provided frequency and forcing amplitude ranges correspond to low energy levels or intervals.

Acknowledgments The authors would like to thank "La Region Auvergne-Rhone-Alpes" for supporting this work in the frame of the CALIPSO project.

\section{References}

[1] George W Housner, Lawrence A Bergman, T Kf Caughey, Anastassios G Chassiakos, Richard O Claus, Sami F Masri, Robert E Skelton, TT Soong, BF Spencer, and James TP Yao. "Structural control: past, present, and future". Journal of engineering mechanics 123.9 (1997), pp. 897-971 (cit. on p. 1).

[2] R.A. Ibrahim. "Recent advances in nonlinear passive vibration isolators". Journal of Sound and Vibration 314.3-5 (2008), pp. 371-452. DOI: 10.1016/j.jsv.2008.01.014 (cit. on p. 1).

[3] Hermann Frahm. "Device for damping vibrations of bodies." Pat. US Patent 989,958. 1911 (cit. on p. 1).

[4] J. P. Den Hartog. Mechanical vibrations. New York, 1956 (cit. on p. 1).

[5] Robert E. Roberson. "Synthesis of a nonlinear dynamic vibration absorber". Journal of the Franklin Institute 254.3 (1952), pp. 205-220. DOI: 10.1016/0016-0032(52)90457-2 (cit. on p. 1).

[6] O. Gendelman, L. I. Manevitch, A. F. Vakakis, and R. M'Closkey. "Energy Pumping in Nonlinear Mechanical Oscillators: Part I-Dynamics of the Underlying Hamiltonian Systems". Journal of Applied Mechanics 68.1 (2001), p. 34. DOI: 10.1115/1.1345524 (cit. on p. 2).

[7] A. F. Vakakis and O. Gendelman. "Energy Pumping in Nonlinear Mechanical Oscillators: Part II. Resonance Capture". Journal of Applied Mechanics 68.1 (2001), p. 42. DOI: $10.1115 / 1.1345525$ (cit. on p. 2).

[8] A. F. Vakakis, ed. Nonlinear targeted energy transfer in mechanical and structural systems I. Solid mechanics and its applications 156. Dordrecht, 2008 (cit. on p. 2).

[9] A. F. Vakakis, ed. Nonlinear targeted energy transfer in mechanical and structural systems II. Solid mechanics and its applications 156. Dordrecht, 2008 (cit. on p. 2). 
[10] O. V. Gendelman, Y. Starosvetsky, and M. Feldman. "Attractors of harmonically forced linear oscillator with attached nonlinear energy sink I: Description of response regimes". Nonlinear Dynamics 51.1 (2008), pp. 31-46. DOI: 10.1007/s11071-006-9167-0 (cit. on pp. 2, 19).

[11] Hiroshi Matsuhisa, Rongrong Gu, Yongjing Wang, Osamu Nishihara, and Susumu Sato. "Vibration Control of a Ropeway Carrier by Passive Dynamic Vibration Absorbers". JSME international journal. Ser. C, Dynamics, control, robotics, design and manufacturing 38.4 (1995), pp. 657-662. DOI: 10.1299/jsmec1993.38.657 (cit. on p. 2).

[12] Hiroshi Matsuhisa and Masashi Yasuda. "Dynamic vibration absorber for pendulum type structure". Pat. US Patent 5,460,099. 1995 (cit. on p. 2).

[13] Y. Starosvetsky and O. V. Gendelman. "Dynamics of a strongly nonlinear vibration absorber coupled to a harmonically excited two-degree-of-freedom system". Journal of Sound and Vibration 312.1 (2008), pp. 234 -256. DOI: 10.1016/j.jsv.2007.10.035 (cit. on p. 2).

[14] Thanh Tung Pham, Claude-Henri Lamarque, and Alireza Ture Savadkoohi. "Multiresonance capturing in a two-degree-of-freedom system under two different harmonic excitations". Journal of Vibration and Control 18.3 (2012), pp. 451-466. DOI: 10.1177/ 1077546311404268 (cit. on p. 2).

[15] L. I. Manevitch. "The Description of Localized Normal Modes in a Chain of Nonlinear Coupled Oscillators Using Complex Variables". Nonlinear Dynamics 25.1 (2001), pp. 95-109. DOI: 10.1023/A:1012994430793 (cit. on p. 5).

[16] Ali Hasan Nayfeh and Dean T. Mook. Nonlinear oscillations. Wiley classics library ed. Wiley classics library. New York, 1995 (cit. on p. 5).

[17] A. Ture Savadkoohi, C.-H. Lamarque, M. Weiss, B. Vaurigaud, and S. Charlemagne. "Analysis of the 1:1 resonant energy exchanges between coupled oscillators with rheologies". Nonlinear Dynamics 86.4 (2016), pp. 2145-2159. DOI: 10.1007/s11071-0162792-3 (cit. on pp. 7, 9).

[18] Y. Starosvetsky and O.V. Gendelman. "Strongly modulated response in forced 2DOF oscillatory system with essential mass and potential asymmetry". Physica D: Nonlinear Phenomena 237.13 (2008), pp. 1719-1733. DOI: 10.1016/j.physd.2008.01.019 (cit. on pp. 7, 16).

[19] C.-H. Lamarque, O. V. Gendelman, A. Ture Savadkoohi, and E. Etcheverria. "Targeted energy transfer in mechanical systems by means of non-smooth nonlinear energy sink". Acta Mechanica 221.1-2 (2011), pp. 175-200. DOI: 10.1007/s00707-011-0492-0 (cit. on p. 19).

[20] Alireza Ture Savadkoohi, Claude-Henri Lamarque, and Zoran Dimitrijevic. "Vibratory energy exchange between a linear and a nonsmooth system in the presence of the gravity". Nonlinear Dynamics 70.2 (2012), pp. 1473-1483. DOI: 10.1007/s11071-0120548-2 (cit. on p. 19).

[21] Oleg V. Gendelman. "Targeted energy transfer in systems with non-polynomial nonlinearity". Journal of Sound and Vibration 315.3 (2008), pp. 732-745. DOI: 10.1016/j. jsv.2007.12.024 (cit. on p. 19). 